\title{
On some topics for the numerical simulation of ductile fracture
}

\author{
P.J. Sánchez ${ }^{\mathrm{a}}$, A.E. Huespe ${ }^{\mathrm{a}, *}$, J. Oliver ${ }^{\mathrm{b}}$

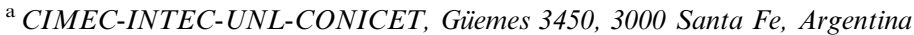 \\ ${ }^{\mathrm{b}}$ E.T.S. Enginyers de Camins, Canals i Ports, Technical University of Catalonia, Campus Nord UPC, \\ Mòdul C1, Gran Capitán s/n, 08034 Barcelona, Spain
}

Received 29 January 2007; received in final revised form 11 August 2007

Available online 24 August 2007

\begin{abstract}
In this work, we analyze some aspects of the macroscopic Gurson-Tvergaard-Needleman (GTN) constitutive model when it is addressed to solve ductile fracture problems by means of numerical simulations:

(i) The analytical solutions of the material discontinuous bifurcation problem is performed. Closed and exact formulas are obtained. The so determined critical conditions and the developed strain localization mode are afterward studied and compared in crack growth problems. Even when this methodology of analysis is rather standard at the present, the conclusions drawn from this study differ significantly from that obtained with a similar analysis in quasi-brittle fracture problems.

(ii) A new very robust numerical integration method for the GTN model, namely the Impl-Ex Method, is proposed. It is a low computational cost algorithm, equivalent to a linear problem per each integration step, with a reasonable precision for engineering purposes. Its accuracy and convergence rate is assessed by means of an error study applied to a ductile fracture test simulation.

(iii) A detailed analysis of a plane strain ductile crack growth problem is performed in a material containing two size-scale of voids. In the analysis, particular attention is given to the mesh size dependence and to the coalescence of the larger void.
\end{abstract}

(C) 2007 Elsevier Ltd. All rights reserved.

\footnotetext{
* Corresponding author. Tel.: +54 342 4511594x1021; fax: +54 3424511169.

E-mail address: ahuespe@intec.unl.edu.ar (A.E. Huespe).
} 
Keywords: (A) Ductile crack growth; (B) Elasto-plastic porous material; (C) Finite elements; Impl-Ex algorithm; Bifurcation and strain localization by void coalescence

\section{Introduction}

Ductile failure, understood as those processes involving localization of plastic strains and ductile crack growth, is a topic that continuously motivates theoretical research lines and numerical developments due to their potential applications in material science, technology and metallurgical industry.

From a micro-mechanical point of view, ductile failure is characterized by three coupled mechanisms: nucleation, growth and coalescence of micro voids, which are induced in the metal alloy matrix by the existence of weaker inclusions. The last mechanism, coalescence, has important consequences at macroscopic level, it induces a notable strain softening at the large-scale material response. Therefore, the material becomes susceptible of bifurcations, instabilities and localized deformation modes.

It was suggested in many previous publications that, in order to predict the constitutive ductile response of a structural component, it is necessary to study, in detail, the evolution of those micro cavities and its influence on the stress state. Otherwise, it is not possible to

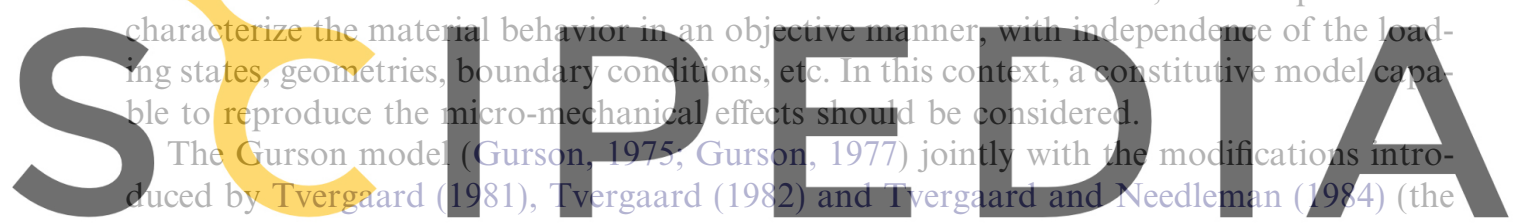

GTN model) is, perhaps, one of the most popular constitutive laws formulated following

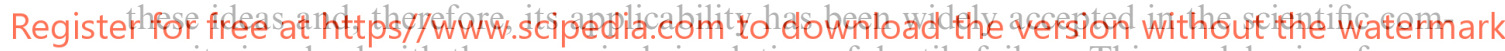
munity involved with the numerical simulation of ductile failure. This model arises from a micro-mechanical analysis assuming the existence of spherical voids embedded in a standard $J_{2}$ elasto-plastic matrix material. The resultant macroscopic law can efficiently simulate both the nucleation and the growth of micro cavities. These two degradation mechanism are considered in the model by means of a scalar damage-like variable, namely the volumetric void fraction " $f$ ", whose evolution governs the gradual loss of material strength. On the other hand, the void coalescence process is a more complex micromechanical effect and constitutes an important limitation of the model response. A possible strategy for including this effect, consists of adopting an empirical criterion where the void coalescence occurs for a predefined (constant) critical value of porosity $f_{\mathrm{C}}$, see for example Needleman and Tvergaard (1987), Xia et al. (1995), Gao et al. (1998), fitting this new parameter by experimental or numerical validation. However, there is no physical evidence assuring the existence of a constant void fraction value which, being independent of the geometry or loading state, determines the coalescence onset. An alternative concept was proposed by Thomason (1985), Thomason (1998). Zhang (1998) used it in order to formulate the so called Complete Gurson Model. Furthermore, it should be mentioned that the coalescence phenomenon depends strongly of the interaction between neighbor voids, see Tvergaard and Hutchinson (2002).

Nevertheless, in the present work, we are not interested neither in a detailed discussion of the fundamental mechanisms governing the ductile crack growth problems nor in a 
comparison between different available constitutive models devised to capture it. Actually, the main objective of this contribution is to present new computational developments addressed to solve numerically that problem.

Although, as a starting assumption, we adopt the classical GTN constitutive model to describe the underlying macroscopic material response and a linear kinematics hypothesis (infinitesimal strains), these aspects do not restrict our final conclusions. The issues addressed in this paper could be straightforwardly extended to cover alternative ductile failure criteria, as well as the exact kinematics.

Two contributions are addressed:

(i) First, we study the standard discontinuous bifurcation analysis applied to the GTN model. The material instability criterion is based on the classical Rice's contribution. In this context, we introduce an analytical strategy capable to solve the problem in a closed form (exactly for the proposed model). This procedure is general, in the sense that it is not restricted to bidimensional cases. It computes the first time that the localization (or acoustic) elasto-plastic tensor becomes singular during the material response history, as well as the direction of the deformation mode compatibles with the bifurcation angle that defines the tensor singularity. The influence of the stress state and stress triaxiality ratio on the critical bifurcation conditions, is studied through a parametric analysis in terms of the mean stress and porosity. Furthermore,

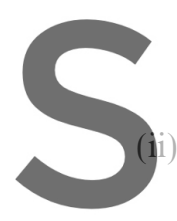
under the conditions this study it is of th are not restricted Time integration new method for th ns that yield stress is
he order $\sigma_{\mathrm{y}} / \mathrm{E} \approx 10^{-}$
to the small strain $\mathrm{k}$
trategies are also ana
e integration of the,
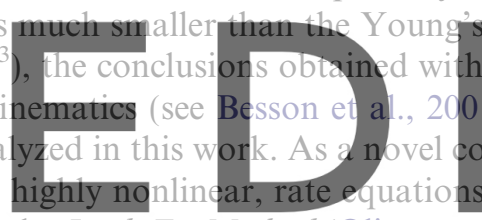

model is proposed. It has been termed the Impl-Ex Method (Oliver et al., 2005). As

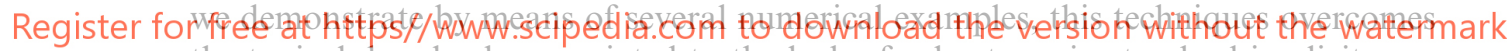
the typical drawbacks associated to the lack of robustness in standard implicit procedures, mainly because the method guarantees the positive definiteness of the consistent tangent constitutive tensor when the strain localization and void-coalescence phenomena become the dominant mechanisms of failure. Additionally, it results a low computational cost strategy with reasonable precision for engineering purposes. Quantitative and qualitative analysis for the new algorithm are presented.

These two mentioned topics are then applied to analyze typical ductile failure situations. Special attention is paid to solve tensile bar tests, in plane strain or axial symmetry cases, and the crack growth problem in a material containing a void population with two different size-scales. We remark, in order to understand the correct scope of the paper, that we address the study of the ductile fracture problem arising as a material instability phenomenon. Problems displaying geometrical instabilities (such as necking due to resistant net section changes) are, obviously, not addressed in the article.

The numerical discrete model has been implemented using a bilinear quadrilateral BBAR finite element formulation, taken from Simo and Hughes (1998). This type of finite element shows good performances for solving solid mechanics problems characterized by presenting isochoric deformations.

The paper is organized as follows: in the next section, the governing rate equations, defining the GTN constitutive law, are introduced. In Section 3, we present a general 
and analytical material instability analysis and obtain closed formulas to determinate the critical condition. Once this condition is fulfilled, the corresponding bifurcation mode could be induced and therefore, the strain localization phenomenon starts. In Section 4, a new integration scheme (Impl-Ex method) applied to the GTN model is formulated. The numerical examples presented in Sections 5 and 6 demonstrate the potential applicability of the Impl-Ex integration algorithm. Important remarks about the correspondence between the first and posterior predicted material bifurcation conditions and the observed localization mode in the solid, are shown. The conclusion drawn from the analysis of this particular point is quite different from that observed in quasi-brittle material failure processes (Oliver et al., 2002; Belytschko et al., 2003). Finally, some conclusions are summarized. In two final appendices, useful expressions can be found.

\section{The GTN model definition}

Unlike the constitutive laws that follow the classic $J_{2}$ criterion, the Gurson model postulates that both components of the stress tensor $\sigma$ : the spherical $(-p \mathbb{1})$ and deviatoric part $(\boldsymbol{S}=\operatorname{dev}(\boldsymbol{\sigma}))$, appear in the definition of the yield surface which, jointly with the plastic flow normality rule, allow for non-isochoric plastic deformations. From contributions proposed by Tvergaard $(1981,1982)$ and Tvergaard and Needleman (1984), the yield condition can be expressed as
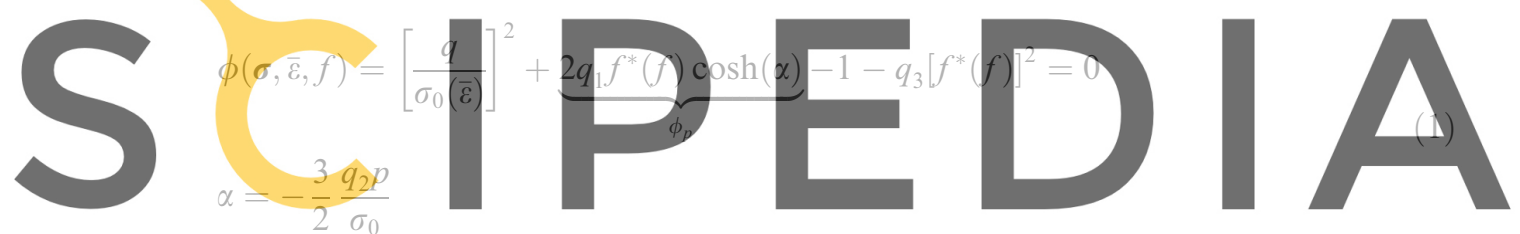

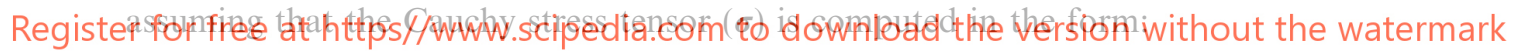

$$
\sigma(p, q)=-p 1+\frac{2}{3} q n ; \quad n=\sqrt{3 / 2}(S /\|S\|)
$$

where $p=-\frac{1}{3} \operatorname{tr}(\boldsymbol{\sigma})$ represents the hydrostatic pressure (minus the mean stress), 1 is the second order identity tensor, $q=\left[\frac{3}{2}(\boldsymbol{S}: S)\right]^{\frac{1}{2}}$ the equivalent stress, $q_{1}, q_{2}$ and $q_{3}$ $\left(q_{3}=q_{1}^{2}\right)$ are material parameters to be estimated by experimental validation and $\sigma_{0}$ is the microscopic yield stress depending (only) on the microscopic equivalent plastic strain $\bar{\varepsilon}$ in the matrix material, through the hardening law:

$$
\sigma_{0}=\sigma_{0}(\bar{\varepsilon})
$$

In order to emphasize the yield function dependence with the pressure, the second term of Eq. (1) is called $\phi_{p}$. In the development of the following numerical algorithm, this term will be subjected to a particular treatment. The void fraction is considered through the scalar magnitude $f$ which acts as an additional internal variable of the model. Its evolution induces, at macroscopic or structural level, the material softenig behavior. Following Tvergaard and Needleman (1984), the void-coalescence phenomenon can be considered by means of a modified void fraction function $f^{*}$ in the definition of the yield criterion:

$$
f^{*}= \begin{cases}f & \text { for } f \leqslant f_{\mathrm{C}} \\ f_{\mathrm{C}}+\frac{\frac{1}{q_{1}}-f_{\mathrm{C}}}{f_{\mathrm{F}}-f_{\mathrm{C}}}\left(f-f_{\mathrm{C}}\right) & \text { for } f_{\mathrm{C}}<f<f_{\mathrm{F}}\end{cases}
$$


where $f^{*}\left(f_{\mathrm{F}}\right)=\frac{1}{q_{1}}$ is the maximum admissible porous fraction value compatible with Eq. (1). The modification (4) is activated when $f>f_{\mathrm{C}}$, being $f_{\mathrm{C}}$ the critical level of the void fraction that induces the coalescence process, whereas the complete degradation is reached for $f_{\mathrm{F}}$ (i.e. $\phi=0$, such that $p=0$ and $q=0$ ). In this work it is assumed: $f_{\mathrm{C}}=0.15$ and $f_{\mathrm{F}}=0.25$.

Considering linear kinematics, it is valid the additive decomposition of the macroscopic strain tensor $(\varepsilon)$ in terms of a reversible elastic component $\left(\varepsilon^{\mathrm{e}}\right)$ and a plastic irreversible counterpart $\left(\varepsilon^{\mathrm{p}}\right)$ :

$$
\boldsymbol{\varepsilon}=\boldsymbol{\varepsilon}^{\mathrm{e}}+\boldsymbol{\varepsilon}^{\mathrm{p}}
$$

The macroscopic plastic strain rate follows the classical normality assumption. It is expressed as

$$
\dot{\boldsymbol{\varepsilon}}^{\mathrm{p}}=\gamma \frac{\partial \phi}{\partial \sigma}=\gamma \boldsymbol{M}
$$

where $\gamma$ is the plastic multiplier and $\boldsymbol{M}$ its direction tensor, showing that the inelastic strain rate field $\left(\dot{\varepsilon}^{\mathrm{p}}\right)$ results orthogonal to the yield surface in the stress space. In view of expression (2), Eq. (6) can be rewritten in terms of the volumetric and deviatoric component of the plastic strain tensor:
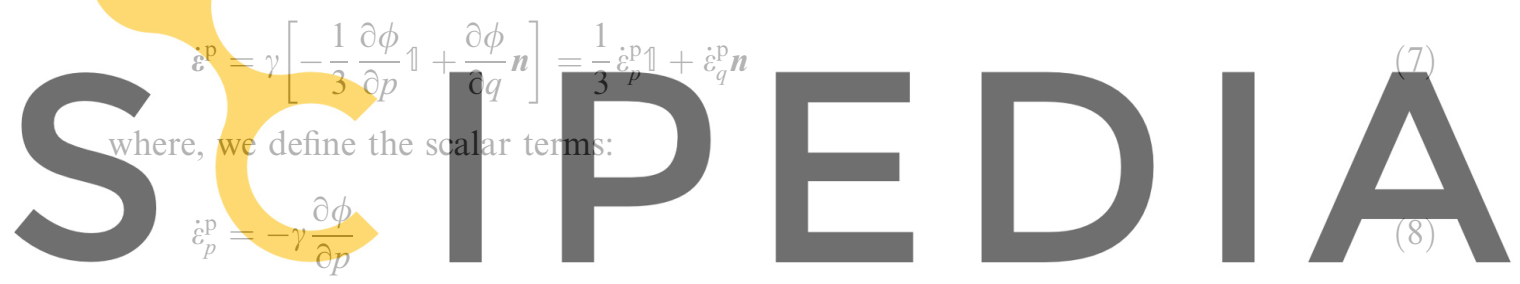

Register for free' at https//www.scipedia.com to download the version without the watermark

From Eqs. (8), (9), it trivialiy follows:

$$
\dot{\varepsilon}_{p}^{\mathrm{p}} \frac{\partial \phi}{\partial q}+\dot{\varepsilon}_{q}^{\mathrm{p}} \frac{\partial \phi}{\partial p}=0
$$

Following Tvergaard (1990), we consider two terms that contribute to the porous fraction evolution law. The first one, associated to the void growth rate, depends strongly on the stress triaxiality state $(T=-p / q)$ through the trace of $\dot{\boldsymbol{\varepsilon}}^{\mathrm{p}}\left(\dot{\varepsilon}_{p}^{\mathrm{p}}=\operatorname{tr}\left(\dot{\boldsymbol{\varepsilon}}^{\mathrm{p}}\right)\right)$. The remaining term simulates the void nucleation rate and it turns out to be a function of the microscopic equivalent plastic strain $(\dot{\bar{\varepsilon}})$ in the matrix material:

$$
\dot{f}=\underbrace{(1-f) \dot{\varepsilon}_{p}^{\mathrm{p}}}_{\text {growth }}+\underbrace{\mathscr{A}_{m}(\bar{\varepsilon}) \dot{\bar{\varepsilon}}}_{\text {nucleation }}
$$

where the $A_{m}(\bar{\varepsilon})$ function is defined as a normal distribution law with mean and standard deviation values given by $\bar{\varepsilon}_{\mathrm{N}}$ and $s_{\mathrm{N}}$ respectively, see Chu and Needleman (1980):

$$
\mathscr{A}_{m}=\mathscr{A}_{m}(\bar{\varepsilon})=\frac{f_{\mathrm{N}}}{s_{\mathrm{N}} \sqrt{2 \pi}} \exp \left[-\frac{1}{2}\left(\frac{\bar{\varepsilon}-\bar{\varepsilon}_{\mathrm{N}}}{s_{\mathrm{N}}}\right)^{2}\right]
$$


Postulating the balance of plastic work rate between the micro $\left(\sigma_{0}, \dot{\bar{\varepsilon}}\right)$ and macroscopic $\left(\boldsymbol{\sigma}, \dot{\boldsymbol{\varepsilon}}^{\mathrm{p}}\right)$ magnitudes, it is obtained the following equation which governs the evolution of the microscopic equivalent plastic strain:

$$
\dot{\bar{\varepsilon}}=\frac{\boldsymbol{\sigma}: \dot{\boldsymbol{\varepsilon}}^{\mathrm{p}}}{(1-f) \sigma_{0}}
$$

Finally, the complementarity (loading-unloading) conditions are introduced as follows:

$$
\phi(p, q, \bar{\varepsilon}, f) \leqslant 0 ; \quad \gamma \geqslant 0 ; \quad \gamma \phi(p, q, \bar{\varepsilon}, f)=0
$$

\section{Material bifurcation analysis}

In this section we analyze the classical problem of material discontinuous bifurcation (Rudnicki et al., 1975; Rice, 1976). Particularly, a closed formula to detect the critical condition is shown. The solution of this problem gives the first possible bifurcation mode, although, as we will show in the following sections, generally it does not correspond with the strain localization mechanism which is finally activated.

Initially, the tangent constitutive modulus of the GTN model ( $\boldsymbol{C}$ ) must be determined. Subsequently, the analytical expression of the bifurcation condition is derived. The final subsection shows an application of this issue.
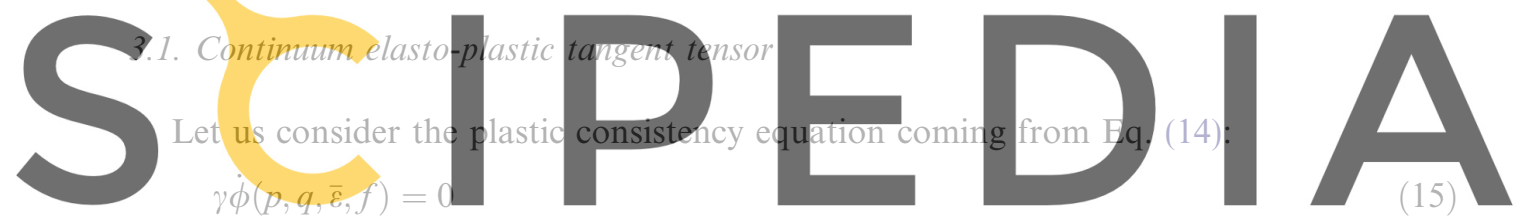

Register for free at https///www.scipedia.com to downlogd the version without the watermark

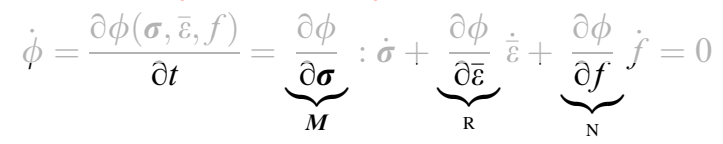

where $\boldsymbol{M}, \mathrm{N}$ and $\mathrm{R}$ are defined in Appendix A.2.

The stress-strain incremental relation and the flow rule determine the following equality:

$$
\dot{\boldsymbol{\sigma}}=\boldsymbol{C}^{\mathrm{e}}:\left(\dot{\boldsymbol{\varepsilon}}-\dot{\boldsymbol{\varepsilon}}^{\mathrm{p}}\right)=\boldsymbol{C}^{\mathrm{e}}:(\dot{\boldsymbol{\varepsilon}}-\gamma \boldsymbol{M})
$$

where $C^{\mathrm{e}}$ is the elastic isotropic constitutive Hooke's tensor, see Eq. (A.23) in Appendix A.2.

Substituting (17), (11) and (13) in (16), the plastic multiplier $\gamma$ could be obtained:

$$
\gamma=\frac{\left(\boldsymbol{M}: \boldsymbol{C}^{\mathrm{e}}\right)}{\xi(H)}: \dot{\boldsymbol{\varepsilon}}=\frac{\boldsymbol{P}}{\xi(H)}: \dot{\boldsymbol{\varepsilon}}
$$

where $\boldsymbol{P}=\left(\boldsymbol{M}: \boldsymbol{C}^{\mathrm{e}}\right)$. The scalar term $\xi(H)$, which depends on the material hardening modulus

$$
H=\frac{\partial \sigma_{0}}{\partial \bar{\varepsilon}}
$$


is given by the following expression:

$$
\xi(H)=\left(\boldsymbol{M}: \boldsymbol{C}^{\mathrm{e}}: \boldsymbol{M}\right)-\mathrm{N}(1-f) \operatorname{tr}(\boldsymbol{M})-\left(\mathrm{N} \mathscr{A}_{m}+\mathrm{R}\right) \frac{(\boldsymbol{\sigma}: \boldsymbol{M})}{(1-f) \sigma_{0}}
$$

After inserting Eq. (18) in (17), it is obtained the loading tangent modulus:

$$
\begin{aligned}
& \dot{\boldsymbol{\sigma}}=\boldsymbol{C}^{\mathrm{ep}}: \dot{\boldsymbol{\varepsilon}} \\
& \boldsymbol{C}^{\mathrm{ep}}=\boldsymbol{C}^{\mathrm{e}}-\frac{\left(\boldsymbol{C}^{\mathrm{e}}: \boldsymbol{M}\right) \otimes\left(\boldsymbol{M}: \boldsymbol{C}^{\mathrm{e}}\right)}{\xi(H)}=\boldsymbol{C}^{\mathrm{e}}-\frac{\boldsymbol{P} \otimes \boldsymbol{P}}{\xi(H)}
\end{aligned}
$$

When $f \neq 0$, it is not possible to assure the $C^{\mathrm{ep}}$ strong ellipticity character even if $H>0$, which means that the model could be subjected to material instability, bifurcation and strain localized modes.

The general format of the tangent constitutive modulus $(\boldsymbol{C})$ can be written as follows:

$$
\dot{\sigma}=\mathrm{C}: \dot{\varepsilon} ; \quad \mathbf{C}= \begin{cases}\mathbb{C}^{\mathrm{C}} & \text { if } \gamma=0 \text { (elastic unloading) } \\ \mathbf{C}^{\mathrm{ep}} & \text { if } \gamma>0 \text { (plastic loading) }\end{cases}
$$

\subsection{Analytical (closed) solution of the material bifurcation problem}

The critical conditions which can induce a "feasible material bifurcation mode" depend

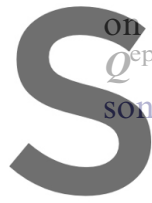

the spectral proper

$=\eta \cdot C^{\mathrm{e}} \cdot \eta$, where $\boldsymbol{\eta}$ is a

(1991), in particular

$\operatorname{det}\left[Q^{\mathrm{ep}}(H, \eta, t)\right]=\operatorname{det}$
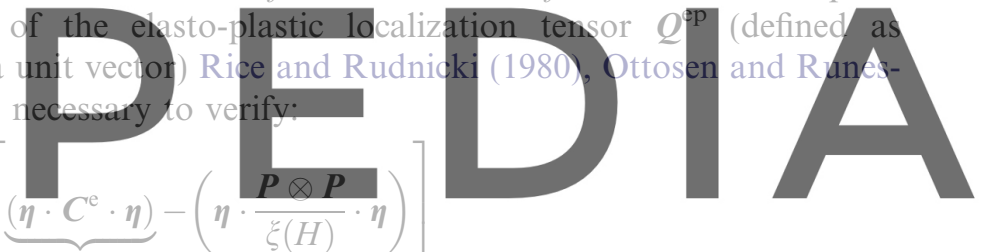

Register for free at https//www.scipedia.com to download the version without the watermark

$$
=\underbrace{\operatorname{det}\left(Q^{\mathrm{e}}\right)}_{>0}\left[1-\frac{Z(H, \eta, t)}{\xi(H, t)}\right]=0
$$

note that $\boldsymbol{Q}^{\mathrm{e}}$ is the elastic acoustic tensor, see Eq. (A.26) in Appendix A.2.

In Eq. (24), $\boldsymbol{\eta}$ represents the unit vector being orthogonal to the strain localization direction, $t$ the (pseudo) time of analysis and $Z(H, \boldsymbol{\eta}, t)$, assuming isotropic elasticity (see definition of $\boldsymbol{C}^{\mathrm{e}}$ in Eq. (A.23)), is a function that can be evaluated as

$$
Z(H, \boldsymbol{\eta}, t)=\boldsymbol{\eta} \cdot \boldsymbol{P} \cdot \boldsymbol{Q}^{\mathrm{e}-1} \cdot \boldsymbol{P} \cdot \boldsymbol{\eta}=\frac{(\boldsymbol{\eta} \cdot \boldsymbol{P} \cdot \boldsymbol{P} \cdot \boldsymbol{\eta})}{\mu}-\frac{(\boldsymbol{\eta} \cdot \boldsymbol{P} \cdot \boldsymbol{\eta})^{2}}{2 \mu(1-\mu)}
$$

Classically, the material instability criterion is associated to finding those conditions such that Eq. (24) is fulfilled for the first time. This situation can be mathematically formulated by means of a constrained minimization procedure. We can express it as follows (see Oliver and Huespe (2004)): given a hardening modulus $H$, find $\boldsymbol{\eta}^{\text {crit }}$ (the critical direction) such that:

$$
\boldsymbol{\eta}^{\text {crit }}=\arg \left\{\min _{\|\boldsymbol{\eta}\|=1} \operatorname{det}\left[\boldsymbol{Q}^{\mathrm{ep}}(H, \boldsymbol{\eta}, t)\right]\right\}
$$

then, the (first) bifurcation time, $t=t_{B}$, results when

$$
\operatorname{det}\left[\boldsymbol{Q}^{\mathrm{ep}}\left(H, \boldsymbol{\eta}^{\mathrm{crit}}, t=t_{B}\right)\right]=0
$$


From Eqs. (24) and (27), we can formulate a criterion to detect material bifurcation $\left(t_{B}\right)$ :

$$
\begin{aligned}
& \text { while } \xi(H)>Z^{\text {crit }}\left(H, \boldsymbol{\eta}^{\text {crit }}, t\right) \Rightarrow t<t_{B} \Rightarrow \operatorname{det}\left[\boldsymbol{Q}^{\text {ep }}\left(H, \boldsymbol{\eta}^{\text {crit }}, t\right)\right]>0 \\
& \text { when } \xi(H)=Z^{\text {crit }}\left(H, \boldsymbol{\eta}^{\text {crit }}, t\right) \Rightarrow t=t_{B} \Rightarrow \operatorname{det}\left[\boldsymbol{Q}^{\text {ep }}\left(H, \boldsymbol{\eta}^{\text {crit }}, t\right)\right]=0
\end{aligned}
$$

where the $Z^{\text {crit }}$ value arises after evaluating Eq. (25) with $\boldsymbol{\eta}=\boldsymbol{\eta}^{\text {crit }}$.

In view of expression (24), taking into account that $\xi(H)$ does not depend on $\boldsymbol{\eta}$, Eq. (26) implies necessarily that:

$$
\begin{aligned}
& \boldsymbol{\eta}^{\text {crit }}=\arg \left\{\max _{\|\boldsymbol{\eta}\|=1} Z(H, \boldsymbol{\eta}, t)\right\} \\
& Z^{\text {crit }}=Z\left(H, \boldsymbol{\eta}^{\text {crit }}, t\right)
\end{aligned}
$$

In order to solve this constrained (maximization) problem, we adopt the geometric strategy proposed by Willam (2000) and Oliver and Huespe (2004). In the last reference, it is demonstrated that a general form for the critical direction $\eta^{\text {crit }}$, lying in the plane defined by the two eigenvectors $\left(\boldsymbol{P}_{\mathrm{I}}, \boldsymbol{P}_{\mathrm{III}}\right)$ of the tensor $\boldsymbol{P}$, could be computed as

$$
\eta^{\text {crit }}=\cos \left(\theta^{\text {crit }}\right) \boldsymbol{P}_{\mathrm{I}}+\sin \left(\theta^{\text {crit }}\right) \boldsymbol{P}_{\mathrm{III}}
$$

in terms of the eritical angle $\theta^{\text {crit }}$ between the first principal direction of tensor $\boldsymbol{P}\left(\boldsymbol{P}_{\mathrm{I}}\right)$ and $\eta^{\mathrm{c}}$

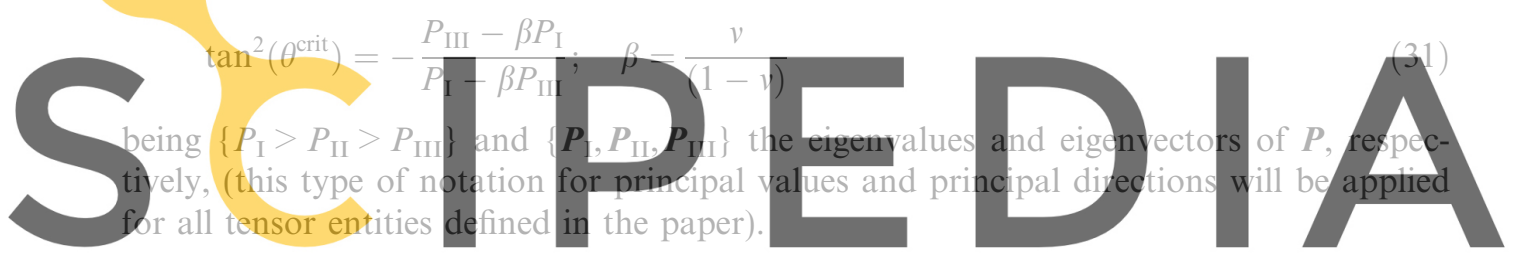

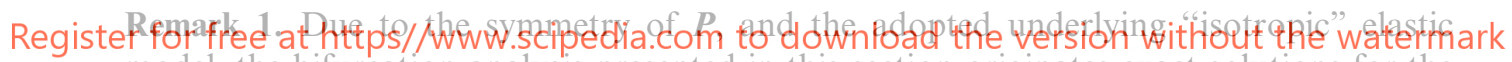
model, the bifurcation analysis presented in this section originates exact solutions for the GTN model, see Oliver and Huespe (2004) for a more detailed discussion about this topic.

Remark 2. Notice that $\boldsymbol{P}$ and $\boldsymbol{\sigma}$ diagonalize in the same orthonormal base, i.e. comparing Eqs. (2) and (A.27), it is verified the coaxiality between their eigenvectors: $\left\{\boldsymbol{P}_{i}\right\} \equiv\left\{\boldsymbol{\sigma}_{i}\right\}$, then $\theta^{\text {crit }}$ could also be interpreted as the angle between the normal vector to the localization band $\left(\boldsymbol{\eta}^{\text {crit }}\right)$ and the first stress principal direction $\sigma_{\mathrm{I}}$.

\subsection{Application}

From the previous analytical study, based on an adequate comparison between the magnitude $\xi(H)$ and the critical value $Z^{\text {crit }}$, it was proven that once the hardening modulus $H$ has been defined (as a material data property), both the stress state $(\boldsymbol{\sigma})$ and the level of void fraction in the matrix material $(f)$ play a role in the determination of the bifurcation conditions. In order to evidence this dependence, we present a series of graphics in the " $p-f$ " space (pressure-porosity). It should be considered that, given each couple $(p, f)$ of points, by using the equation $\phi=0$ and assuming $\bar{\varepsilon}$ fixed, we can parameterize $q(p, f)$ as a function of $p$ and $f$. Therefore, in the plane $(p, f)$, exists an admissible zone of points where $\phi=0$, and a not admissible zone where $\phi>0$. Furthermore, every point $(p, f)$, in 
the admissible zone, has assigned a stress triaxiality factor $T=-p / q$, as it is shown in Fig. $1 b$.
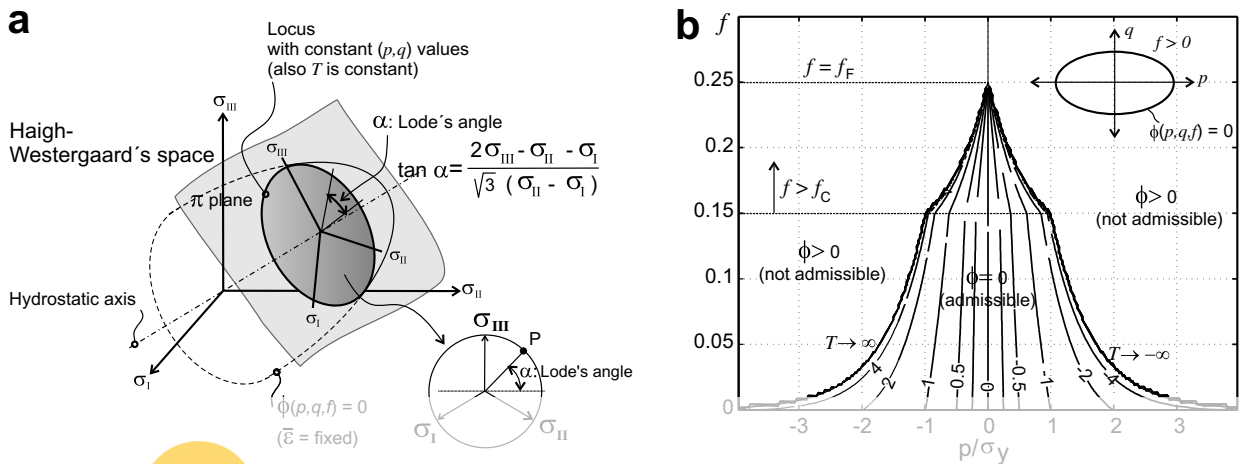

Fig. 1. (a) Yield function representation for the GTN model in the principal stress space. (b) Contour levels of triaxiality ratio: $T=-p / q$.
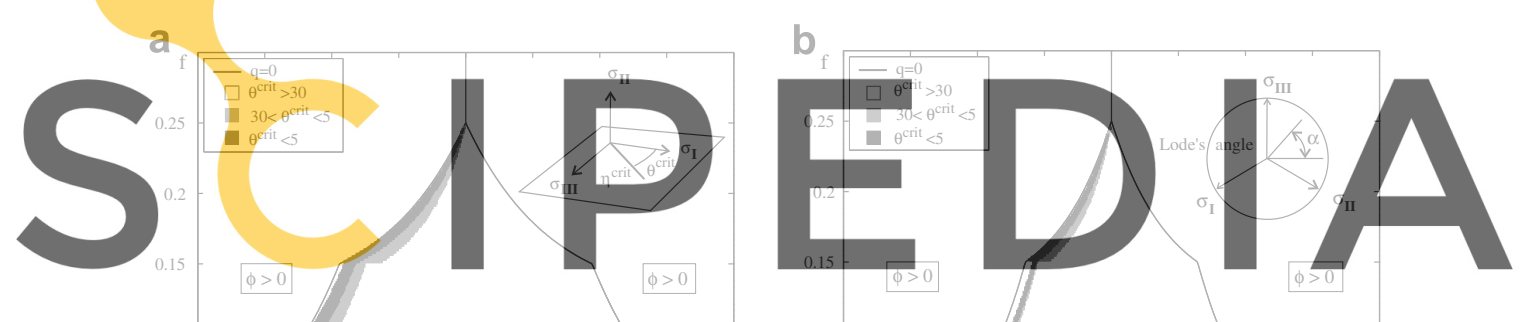

Register for free at https//www.scipedia.com to dovwnload theversion withqut the watermark
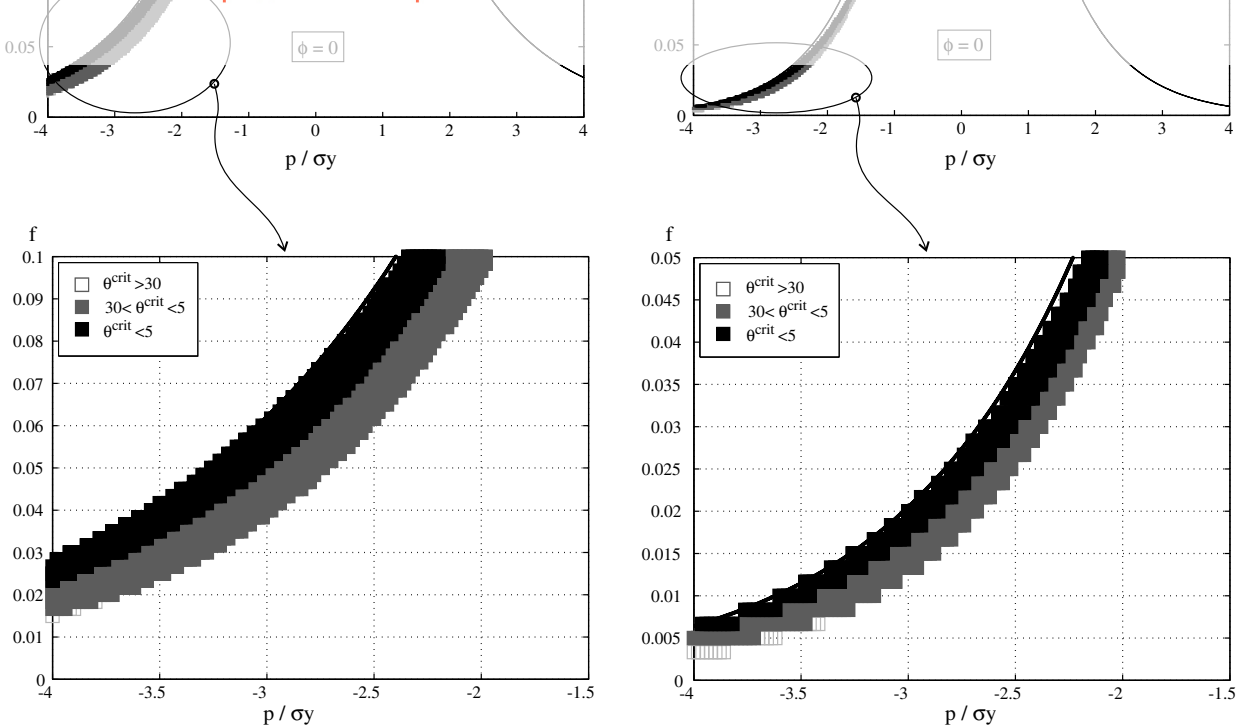

Fig. 2. Material bifurcation analysis for the GTN model assuming a Lode's angle $\alpha=30^{\circ}$ : (a) $\bar{\varepsilon}=2.00, H \approx 0$, $\sigma_{0}=E / 160$. (b) $\bar{\varepsilon}=0.04, H=E / 100, \sigma_{0}=E / 230$. 
In this application, the constitutive material response was characterized by setting the following mechanical parameters: $q_{1}=1.5, q_{2}=1.0, q_{3}=2.25, f_{\mathrm{N}} \overline{\bar{N}} 0.04, s_{\mathrm{N}}=0.1$, $\varepsilon_{\mathrm{N}}=0.3, \sigma_{\mathrm{y}} / E=1 / 300, v=0.3, f_{\mathrm{C}}=0.15, f_{\mathrm{F}}=0.25$ and $\sigma_{0}=\sigma_{\mathrm{y}}\left[1+\frac{E \bar{\varepsilon}}{\sigma_{\mathrm{y}}}\right]^{N}$ with $N=0.1$.

Figs. 2 and 3 plot those points $(p, f)$ satisfying the material instability condition (Eq. (27)). We consider two arbitrary class of deviatoric tensors " $\boldsymbol{n}$ " (see Eq. (2)) defined by their corresponding Lode's angle $(\alpha)$. This angle is directly related to the classical Lode's parameter: $\Xi=-\sqrt{3} \tan \alpha$ (see Hill, 1950, pp. 10) giving the angular position of a stress point $\mathrm{P}$, in the octahedral plane, respect to the horizontal direction, as displayed in Fig. 2a.

In first place, Fig. 2 displays the obtained analytical bifurcation results for $\alpha=30^{\circ}$ and considering two limit situations in terms of the material hardening modulus: $\bar{\varepsilon}=0.04$ determining a high $H$ value $\left(H=E / 100, \sigma_{0}=E / 230\right.$, see Fig. $\left.2 b\right)$ and $\bar{\varepsilon}=2.00$ inducing a low $H$ value $\left(H \approx 0, \sigma_{0}=E / 160\right.$, see Fig. $\left.2 \mathrm{a}\right)$. The microscopic yield stress $\sigma_{0}$ and the hardening modulus $H$, for each case, are computed once defined $\bar{\varepsilon}$ (which is assumed as constant for the complete analysis) following the above defined exponential law. In Fig. 3, the same study is presented but now assuming $\alpha=0^{\circ}$. The gray color scale used

a

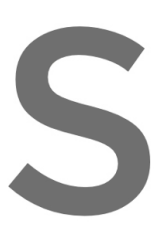

Register for free at https//www.scipedia.com to do
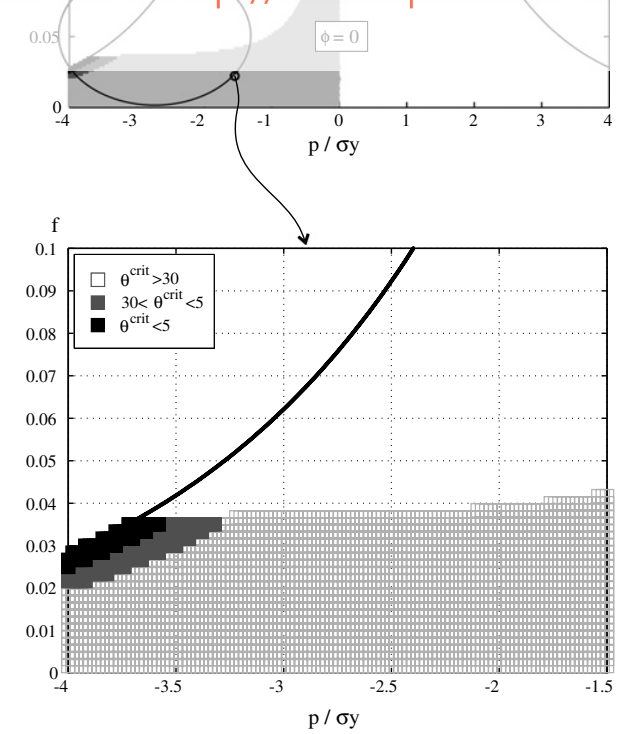

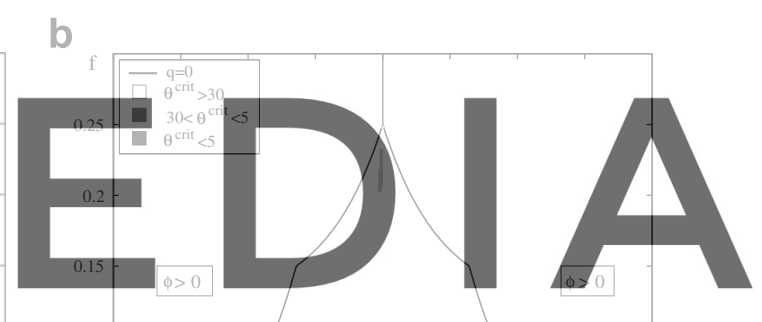

Fig. 3. Material bifurcation analysis for the GTN model assuming a Lode's angle $\alpha=0^{\circ}$ : (a) $\bar{\varepsilon}=2.00, H \approx 0$, $\sigma_{0}=E / 160$. (b) $\bar{\varepsilon}=0.04, H=E / 100, \sigma_{0}=E / 230$.

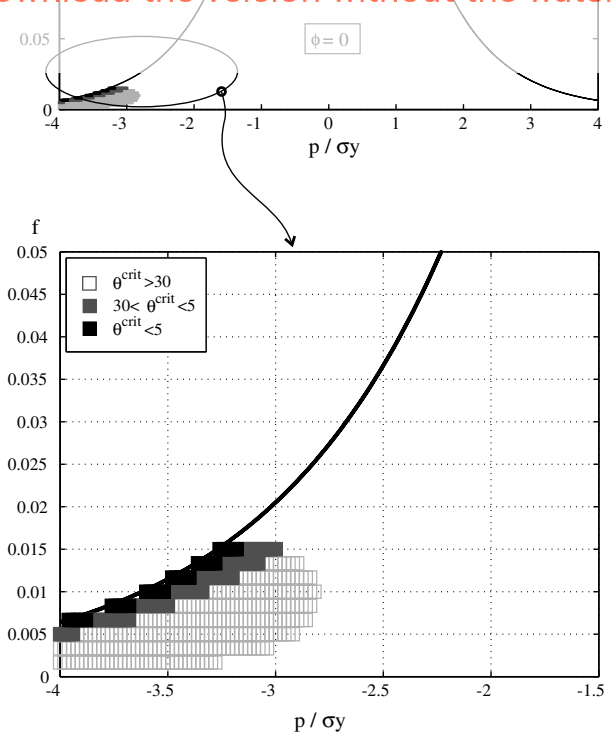


in the graphs (2), (3) establishes a range of values for the critical bifurcation angle $\theta^{\text {crit }}$, computed from Eq. (31).

In view of the previously shown results, we can conclude that the GTN model is able to predict different bifurcation modes. In this sense, it must be emphasized that the stress triaxiality ratio plays an important role in the obtained bifurcation angle. As $T$ increases $(T \rightarrow \infty)$, the void growth term in the evolution law of $f$ (Eq. (11)) becomes more and more significant, then the yield function displays a greater curvature evidencing a marked difference with respect to the classical Von Mises model. In that case, the bifurcation study yields a $\boldsymbol{\eta}^{\text {crit }}$ vector tending to be collinear with $\boldsymbol{\sigma}_{\mathrm{I}}\left(\theta^{\text {crit }} \approx 0^{\circ}\right)$ and consequently a mode I of failure could be induced. Two special remarks can be done: (i) the angle $\theta^{\text {crit }} \approx 0^{\circ}$ is only observed when high stress triaxiality values are present; (ii) $\theta^{\text {crit }} \approx 0^{\circ}$ is not possible for $J_{2}$ based constitutive laws which postulate, invariably, shear bands type failure mechanisms $\left(\theta^{\text {crit }} \approx 45^{\circ}\right)$.

It can be observed, from Figs. 1-3, the modification introduced by the adopted coalescence criterion when the void fraction reaches the critical value $f=f_{\mathrm{C}}$, see Eq. (4).

Remark 3. Considering $f=0$, the $\phi$ function becomes identical to the yield criterion for the $J_{2}$ standard plasticity model, however Eq. (20) jointly with Eq. (22) show that, even in such special case, the constitutive tangent tensors are not equivalent for both models, because the terms $\mathrm{N}$ and $\mathscr{A}_{m}$ do not necessarily vanish. This situation explains the differences between Figs. 2a and 3a, for $f=0$ (abscissa axis), even when they only differ by the Lode's angle definition, being that the bifurcation analysis applied to $J_{2}$ model does not depend on the Lode's angle.

\section{Numerical integration}

Different methodologies addressed to integrate the GTN constitutive model have been presented in the literature, see for example Zhang (1995), Kojic et al. (2002), Betegon et al. (2006), among others. In the general case, the coupled equation system determined by an implicit Euler-Backward method cannot be solved by means of a standard Newton-Raphson scheme because, due to the highly nonlinear evolution of the internal variables, the convergence fails very frequently. Aravas (1987) has presented a strategy based on the partition of the equation system and a double loop to improve the robustness of the standard implicit method.

It should be mentioned that, in all cases where material instabilities are present, the strategies based on implicit procedures yield consistent stiffness matrices that become not positive definite (a singular point in the fundamental equilibrium branch is crossed) in some stage of the softening regime, irrespective of the control method (arc-length) to be used in the time integration procedure. Obviously, this feature introduce a serious drawback in the simulation of problems, becoming a source of numerical difficulties (Oliver and Huespe, 2004).

In Oliver et al. (2005) a time integration algorithm based on an implicit-explicit (ImplEx) strategy was recently proposed. In that reference it was shown the benefits and good performances obtained when this technique is addressed to integrate isotropic damage and $J_{2}$ elasto-plastic models. In the present work, we add some new ingredients to this procedure in order to solve a pressure dependent law, such as the GTN model. 
The Impl-Ex strategy requires a double stress integration per time step. One, based on a standard implicit scheme and the other based on an explicit extrapolation algorithm.

Therefore, in the next subsection, we present an implicit integration scheme based on the Aravas' method. After that, the explicit integration stage is described jointly with the effective tangent tensor consistent with this procedure.

\subsection{Implicit (two-stages) integration scheme}

Applying an Euler-Backward finite difference scheme (implicit integrator) to the algebraic-differential equation system describing the GTN model evolution (normality rule (10), yield condition (1) and evolution laws for internal variables according to Eqs. (13) and (11)), and assuming that a loading process happens, the discrete equations at the integration step $(n+1)$, can be written as

$$
\begin{aligned}
& \Delta \varepsilon_{p}^{\mathrm{p}} \frac{\partial \phi}{\partial q}+\Delta \varepsilon_{q}^{\mathrm{p}} \frac{\partial \phi}{\partial p}=0 \\
& {\left[\frac{q}{\sigma_{0}}\right]^{2}+2 q_{1} f^{*} \cosh (\alpha)-1-q_{3}\left(f^{*}\right)^{2}=0} \\
& \frac{-p \Delta \varepsilon_{p}^{\mathrm{p}}+q \Delta \varepsilon_{q}^{\mathrm{p}}}{(1-f) \sigma_{0}}-\Delta \bar{\varepsilon}=0 \\
& (1-f) \Delta \varepsilon_{p}^{\mathrm{p}}+\mathscr{A}_{m} \Delta \bar{\varepsilon}-\Delta f=0
\end{aligned}
$$

where $\left[\Delta \varepsilon_{p}^{\mathrm{p}}, \Delta \varepsilon_{q}^{\mathrm{p}}, \Delta \bar{\varepsilon}, \Delta f\right]$ represent the finite increments for the internal variable rates $\left[\dot{\varepsilon}_{p}^{\mathrm{p}}, \dot{\varepsilon}_{q}^{\mathrm{p}}, \dot{\bar{\varepsilon}}, \dot{f}\right]$ and all the non-incremental terms are evaluated at time step $(n+1)$. In a more compact form, Eq. (32) can be expressed as $\boldsymbol{R}_{n+1}=\mathbf{0}$, where the residue $(\boldsymbol{R})$ of the equation system is defined by

$$
\boldsymbol{R}=\left[\begin{array}{l}
R_{a} \\
R_{b} \\
\ldots \\
R_{c} \\
R_{d}
\end{array}\right]=\left[\begin{array}{l}
\boldsymbol{R}_{1} \\
\ldots \\
\boldsymbol{R}_{2}
\end{array}\right]=\left[\begin{array}{c}
\Delta \varepsilon_{p}^{\mathrm{p}} \frac{\partial \phi}{\partial q}+\Delta \varepsilon_{q}^{\mathrm{p}} \frac{\partial \phi}{\partial p} \\
\left(\frac{q}{\sigma_{0}}\right)^{2}+2 q_{1} f^{*} \cosh (\alpha)-1-q_{3}\left(f^{*}\right)^{2} \\
\frac{-p \Delta \varepsilon_{p}^{\mathrm{p}}+q \Delta \varepsilon_{q}^{\mathrm{p}}}{(1-f) \sigma_{0}}-\Delta \bar{\varepsilon} \\
(1-f) \Delta \varepsilon_{p}^{\mathrm{p}}+\mathscr{A}_{m} \Delta \bar{\varepsilon}-\Delta f \ldots \ldots \ldots \ldots \ldots \ldots \ldots \ldots \ldots \ldots \ldots \ldots
\end{array}\right]
$$

Note the adopted fractional format for Eq. (33), which will be used to formulate the implicit two-stages procedure.

The solution of system (32) can be found via an iterative procedure. Let the vector $\Delta \boldsymbol{X}=\left[\Delta \varepsilon_{p}^{\mathrm{p}}, \Delta \varepsilon_{q}^{\mathrm{p}}, \Delta \bar{\varepsilon}, \Delta f\right]=\boldsymbol{X}_{n+1}-\boldsymbol{X}_{n}$ represent the finite increments for the internal variables. Then, the iterative first order corrections $\delta \boldsymbol{X}^{(i)}$ for the incremental state variables $\Delta \boldsymbol{X}$ can be computed using the Jacobian matrix $(\boldsymbol{J})$ arising from the linearized form of the equation system (32):

$$
\boldsymbol{J}^{(i)}=\frac{\partial \boldsymbol{R}^{(i)}}{\partial \Delta \boldsymbol{X}}=\left[\begin{array}{ccccc}
J_{a a} & J_{a b} & : & J_{a c} & J_{a d} \\
J_{b a} & J_{b b} & : & J_{b c} & J_{b d} \\
\ldots & \ldots & . & \ldots & \ldots \\
J_{c a} & J_{c b} & : & J_{c c} & J_{c d} \\
J_{d a} & J_{d b} & : & J_{d c} & J_{d d}
\end{array}\right]=\left[\begin{array}{lll}
\boldsymbol{A}_{11} & : & \boldsymbol{A}_{12} \\
\ldots & . & \ldots \\
\boldsymbol{A}_{21} & : & \boldsymbol{A}_{22}
\end{array}\right]
$$


in order to obtain:

$$
\boldsymbol{\delta} \boldsymbol{X}^{(i)}=-\boldsymbol{J}^{(i)^{-1}} \boldsymbol{R}^{(i)} ; \quad \boldsymbol{\delta} \boldsymbol{X}^{(i)}=\left[\begin{array}{c}
\delta \Delta \varepsilon_{p}^{\mathrm{p}} \\
\delta \Delta \varepsilon_{q}^{\mathrm{p}} \\
\ldots \\
\delta \Delta \bar{\varepsilon} \\
\delta \Delta f
\end{array}\right]=\left[\begin{array}{c}
\boldsymbol{\delta}_{1} \\
\ldots \\
\boldsymbol{\delta}_{2}
\end{array}\right]
$$

where the supra-index $(i)$ means iteration number $i$, in the same integration step interval: $(n+1)$. The expressions corresponding to the components of $\boldsymbol{J}$ are derived in Appendix A.1.

In general, the direct application of iteration (35) does not work appropriately to solve (32). Therefore, based in the partition of Eqs. (33) and (34), it has been proposed a double loop fractional (staggered) iterative procedure as shown in Box (1). The internal loop solves $\left\|\boldsymbol{R}_{2}\right\|=0$, by fixing $\boldsymbol{\delta}_{1} \equiv \mathbf{0}$, i.e. $\Delta \varepsilon_{p}^{\mathrm{p}}$ and $\Delta \varepsilon_{q}^{\mathrm{p}}$ remain constant during the loop iterations. Therefore, each iteration determines:

$$
\boldsymbol{\delta}_{2}=\boldsymbol{A}_{22}^{-1} \boldsymbol{R}_{2}
$$

until to the internal loop converges. Based on the equation system (35), the external loop computes $\boldsymbol{\delta}_{1}$, considering $\boldsymbol{R}_{2}=\mathbf{0}$, as follows:

$$
\boldsymbol{\delta}_{1}=\left(\boldsymbol{A}_{11}-\boldsymbol{A}_{12} \boldsymbol{A}_{22}^{-1} \boldsymbol{A}_{21}\right)^{-1} \boldsymbol{R}_{1}
$$

Box 1: Implicit numerical integration algorithm

Integration step: $(n+1)$

INPUT: $\boldsymbol{\varepsilon}_{n+1} ; \boldsymbol{X}_{n}=\left[\boldsymbol{\varepsilon}_{n}^{\mathrm{p}}, \bar{\varepsilon}_{n}, f_{n}\right]$

(1) Trial state

$$
\begin{aligned}
\text { Compute }: \boldsymbol{\sigma}^{\text {trial }} & =\boldsymbol{C}^{\mathrm{e}}:\left(\boldsymbol{\varepsilon}_{n+1}-\boldsymbol{\varepsilon}_{n}^{\mathrm{p}}\right) ; \quad \boldsymbol{S}^{\text {trial }}=\operatorname{dev}\left(\boldsymbol{\sigma}^{\text {trial }}\right) \\
& p_{n+1}=p^{\text {trial }}=-\frac{1}{3} \operatorname{tr}\left(\boldsymbol{\sigma}^{\text {trial }}\right) ; \quad q_{n+1}=q^{\text {trial }}=\left[3 / 2\left(\boldsymbol{S}^{\text {trial }}: \boldsymbol{S}^{\text {trial }}\right)\right]^{1 / 2} \\
\boldsymbol{n}^{\text {trial }} & =\sqrt{3 / 2}\left(\boldsymbol{S}^{\text {trial }} /\|\boldsymbol{S}\|^{\text {trial }}\right) ; \quad \phi^{\text {trial }}=\phi\left(\boldsymbol{\sigma}^{\text {trial }}, \bar{\varepsilon}_{n}, f_{n}\right)
\end{aligned}
$$

Assume: $\Delta \boldsymbol{X}_{n+1}=\mathbf{0}$, i.e $: \Delta \varepsilon_{p}^{\mathrm{p}}=\Delta \varepsilon_{q}^{\mathrm{p}}=\Delta \bar{\varepsilon}=\Delta f=0$

(2) Check yield criterion

If $\phi^{\text {trial }} \leqslant 0 \rightarrow$ Go to step (7)

If $\phi^{\text {trial }}>0 \rightarrow$ Go to step (3)

(3) Newton external loop: iteration for $\Delta \varepsilon_{p}^{\mathrm{p}}$ and $\Delta \varepsilon_{q}^{\mathrm{p}}$, to solve $\boldsymbol{R}_{1}^{n+1}=\mathbf{0}$

Evaluate: $\sigma_{0}=\sigma_{0}(\bar{\varepsilon})$ and $\mathscr{A}_{m}=\mathscr{A}_{m}(\bar{\varepsilon})$ (from Eqs. (3) and (12))

Compute residual: $\boldsymbol{R}_{1}\left(\Delta \varepsilon_{p}^{\mathrm{p}}, \Delta \varepsilon_{q}^{\mathrm{p}}, \Delta \bar{\varepsilon}, \Delta f\right)$, from Eq. (33)

Compute jacobian: $\boldsymbol{J}$, from Eq. (34) (see also Appendix A.1)

Compute increments for the two first equations: $\boldsymbol{\delta}_{1}=\left[\boldsymbol{A}_{11}-\boldsymbol{A}_{12} \boldsymbol{A}_{22}^{-1} \boldsymbol{A}_{21}\right]^{-1} \boldsymbol{R}_{1}$

Update: $\left[\begin{array}{c}\Delta \varepsilon_{p}^{\mathrm{p}} \\ \Delta \varepsilon_{q}^{\mathrm{p}}\end{array}\right] \rightarrow\left[\begin{array}{l}\Delta \varepsilon_{p}^{\mathrm{p}} \\ \Delta \varepsilon_{q}^{\mathrm{p}}\end{array}\right]+\boldsymbol{\delta}_{1}$

Update: $p_{n+1}=p^{\text {trial }}+\kappa \Delta \varepsilon_{p}^{\mathrm{p}}$ and $q_{n+1}=q^{\text {trial }}-3 \mu \Delta \varepsilon_{q}^{\mathrm{p}}$ 
(4) Newton internal loop: iteration for $\Delta \bar{\varepsilon}$ and $\Delta f$, to solve $\boldsymbol{R}_{2}^{n+1}=\mathbf{0}$

valuate: $\sigma_{0}=\sigma_{0}(\bar{\varepsilon})$ and $\mathscr{A}_{m}=\mathscr{A}_{m}(\bar{\varepsilon})$ (from Eqs. (3) and (12))

Compute residual: $\boldsymbol{R}_{2}\left(\Delta \varepsilon_{p}^{\mathrm{p}}, \Delta \varepsilon_{q}^{\mathrm{p}}, \Delta \bar{\varepsilon}, \Delta f\right)$, from Eq. (33)

Compute: $\boldsymbol{A}_{22}$, from Eq. (34) (see also Appendix A.1)

Compute increments for the last two equations: $\boldsymbol{\delta}_{2}=\boldsymbol{A}_{22}^{-1} \boldsymbol{R}_{2}$

Update: $\left[\begin{array}{l}\Delta \bar{\varepsilon} \\ \Delta f\end{array}\right] \rightarrow\left[\begin{array}{c}\Delta \bar{\varepsilon} \\ \Delta f\end{array}\right]+\boldsymbol{\delta}_{2}$

Update: $\bar{\varepsilon}_{n+1}=\bar{\varepsilon}_{n}+\Delta \bar{\varepsilon}$ and $f_{n+1}=f_{n}+\Delta f$

(5) Check convergence for internal loop

Update residual: $\boldsymbol{R}_{2}\left(\Delta \varepsilon_{p}^{\mathrm{p}}, \Delta \varepsilon_{q}^{\mathrm{p}}, \Delta \bar{\varepsilon}, \Delta f\right)$, from Eq. (33)

If $\left\|\boldsymbol{R}_{2}\right\|<$ tol $\rightarrow$ Go to step (6)

else $\rightarrow$ Go back to step (4)

End internal loop

(6) Check convergence for external loop

Update residual: $\boldsymbol{R}_{1}\left(\Delta \varepsilon_{p}^{\mathrm{p}}, \Delta \varepsilon_{q}^{\mathrm{p}}, \Delta \bar{\varepsilon}, \Delta f\right)$, from Eq. (33)

If $\left\|\boldsymbol{R}_{1}\right\|<$ toll $\rightarrow$ Go to step (7)

else $\rightarrow$ Go back to step (3)

\section{End external loop}

(7) Update stress state

$$
\boldsymbol{\sigma}_{n+1}=-p_{n+1} 1+\frac{2}{3} q_{n+1} \boldsymbol{n}^{\text {trial }}
$$

(8) Update plastic strain tensor

$$
\boldsymbol{\varepsilon}_{n+1}^{\mathrm{p}}=\boldsymbol{\varepsilon}_{n}^{\mathrm{p}}+\frac{1}{3} \Delta \varepsilon_{p}^{\mathrm{p}} \mathbb{1}+\Delta \varepsilon_{q}^{\mathrm{p}} \boldsymbol{n}^{\text {trial }}
$$

OUTPUT: $\boldsymbol{\sigma}_{n+1} ; \boldsymbol{X}_{n+1}=\left[\varepsilon_{n+1}^{\mathrm{p}}, \bar{\varepsilon}_{n+1}, f_{n+1}\right]$

\subsection{Implicit-explicit (Impl-Ex) integration scheme}

A consistent application of the above mentioned numerical scheme at the global or structural level, originates an adequate and sufficiently robust methodology when the mechanical response is governed by quasi-homogeneous plastic deformation patterns, associated with low levels of porous fraction in the matrix material.

However, this methodology exhibits a drastic degradation of the numerical stability in the post-critical regime, mainly when ductile failure induced by the void coalescence and the related effect of strain localization is reached. This behavior is due to the loss of the tangent algorithmic operator positive definiteness, which is caused by the material instabilities in that stage of the process.

This reason motivates a modification of the integration procedure. The new method provides two sequence of variables defining the problem: 
- the sequence called "implicit", denoted with the symbol $(\hat{*})$ over each variable: $\left\{\hat{\boldsymbol{\sigma}}, \hat{\boldsymbol{\varepsilon}}^{\mathrm{p}}, \hat{\gamma}, \hat{f}, \hat{\bar{\varepsilon}}\right\}$.

- the sequence called "explicit", denoted with the symbol $(\tilde{*})$ over each variable: $\left\{\widetilde{\boldsymbol{\sigma}}, \tilde{\boldsymbol{\varepsilon}}^{\mathrm{p}}, \widetilde{\gamma}, \widetilde{f}, \tilde{\bar{\varepsilon}}\right\}$.

The Impl-Ex integration strategy has been implemented as we explain next.

\subsubsection{Proposed numerical algorithm for Impl-Ex method}

Let a strain increment $\Delta \varepsilon_{n+1}$ at the integration step $(n+1)$ be given. The implicit set of variables $\left\{\hat{\boldsymbol{\sigma}}_{n+1}, \hat{\boldsymbol{\varepsilon}}_{n+1}^{\mathrm{p}}, \hat{\gamma}_{n+1}, \hat{f}_{n+1}, \hat{\bar{\varepsilon}}_{n+1}\right\}$, are obtained by applying the algorithm of Box (1). The explicit terms $(n+1)$, of the explicit sequence, are determined by the following procedure:

(1) the implicit internal variables $\hat{f}_{n}, \hat{\bar{\varepsilon}}_{n}$ and the plastic multiplier $\hat{\gamma}_{n}$ of the previous time step $(n)$ are assigned to the corresponding explicit variables of the step $(n+1)$ :

$\tilde{f}_{n+1}=\hat{f}_{n}$

$\tilde{\bar{\varepsilon}}_{n+1}=\hat{\bar{\varepsilon}}_{n}$

$\tilde{\gamma}_{n+1}=\hat{\gamma}_{n} \frac{\Delta t_{n+1}}{\Delta t_{n}}$

where, in Eq. (40) and accounting for possible variations between the integration step lengths. $n+1\left(\Delta t_{n+1}\right)$ and $n\left(\Delta t_{n}\right), \hat{\gamma}_{n}$ has been scaled by the factor $\Delta t_{n+1} / \Delta t_{n}$. Once defined $\tilde{f}_{n+1}$ and $\tilde{\bar{\varepsilon}}_{n+1}$, the terms $\tilde{\sigma}_{0 n+1}$ and $\tilde{f}_{n+1}^{*}$ are determined by means of Eqs. (3), (4) respectively:

$\tilde{f}_{n+1}^{*}=f^{*}\left(\tilde{f}_{n+1}\right)$

$\tilde{\sigma}_{0 n+1}=\sigma_{0}\left(\tilde{\bar{\varepsilon}}_{n+1}\right)$

(2) the explicit stress term is then obtained by means of an Elastic Predictor-Plastic Corrector scheme:

$\tilde{\boldsymbol{\sigma}}_{n+1}=\hat{\boldsymbol{\sigma}}_{n}+\boldsymbol{C}^{e}:\left(\Delta \boldsymbol{\varepsilon}_{n+1}-\Delta \tilde{\boldsymbol{\varepsilon}}_{n+1}^{\mathrm{p}}\right)=\underbrace{\hat{\boldsymbol{\sigma}}^{\text {trial }}}_{\text {Elastic Predictor }}-\underbrace{\boldsymbol{C}^{\mathrm{e}}: \Delta \tilde{\boldsymbol{\varepsilon}}_{n+1}^{\mathrm{p}}}_{\text {Plastic Corrector }}$

the first term, $\hat{\boldsymbol{\sigma}}^{\text {trial }}=\hat{\boldsymbol{\sigma}}_{n}+\boldsymbol{C}^{e}: \Delta \boldsymbol{\varepsilon}_{n+1}$, coincides with the implicit elastic predictor of step $(n+1)$. The plastic corrector considers an explicit plastic strain value $\left(\Delta \tilde{\boldsymbol{\varepsilon}}^{\mathrm{p}}\right)$ :

$\Delta \tilde{\boldsymbol{\varepsilon}}_{n+1}^{\mathrm{p}}=\tilde{\gamma}_{n+1} \frac{\partial \psi\left(\tilde{\boldsymbol{\sigma}}_{n+1}, \tilde{f}_{n+1}, \tilde{\bar{\varepsilon}}_{n+1}\right)}{\partial \boldsymbol{\sigma}}$

where the function $\psi$ is similar to the GTN yield criterion:

$\psi=\left[\frac{q}{\sigma_{0}}\right]^{2}+\psi_{p}-1-q_{3}\left(f^{*}\right)^{2}$

except the pressure dependent term $\psi_{p}$ which is a parabolic approach to $\phi_{p}$ in (1):

$$
\begin{aligned}
& \begin{aligned}
\psi_{p}(p) & =\phi_{p}\left(\hat{p}_{n}\right)+\frac{\partial \phi_{p}\left(\hat{p}_{n}\right)}{\partial p}\left[p-\hat{p}_{n}\right]+\frac{1}{2} \frac{\partial^{2} \phi_{p}\left(\hat{p}_{n}\right)}{\partial p \partial p}\left[p-\hat{p}_{n}\right]^{2} \\
& =\phi_{p}\left(\hat{p}_{n}\right)+\tilde{\chi} \tilde{\omega} \sinh \left(\tilde{\omega} \hat{p}_{n}\right)\left[p-\hat{p}_{n}\right]+\frac{1}{2} \tilde{\chi} \tilde{\omega}^{2} \cosh \left(\tilde{\omega} \hat{p}_{n}\right)\left[p-\hat{p}_{n}\right]^{2}
\end{aligned} \\
& \tilde{\chi}=2 q_{1} \tilde{f}_{n+1}^{*} ; \quad \tilde{\omega}=-\frac{3}{2} \frac{q_{2}}{\tilde{\sigma}_{0 n+1}}
\end{aligned}
$$


From Eqs. (43)-(46), the explicit stress $\tilde{\boldsymbol{\sigma}}_{n+1}$ is determined in closed form:

$$
\begin{aligned}
& \tilde{\boldsymbol{\sigma}}_{n+1}=\widetilde{\boldsymbol{S}}_{n+1}-\tilde{p}_{n+1} \mathbb{1} \\
& \widetilde{\boldsymbol{S}}_{n+1}=\left[1+\frac{6 \mu \tilde{\gamma}_{n+1}}{\tilde{\sigma}_{0 n+1}^{2}}\right]^{-1} \hat{\boldsymbol{S}}^{\text {trial }} \\
& \tilde{p}_{n+1}=\left(1+\kappa \tilde{\gamma}_{n+1} \tilde{\chi} \tilde{\omega}^{2} \cosh \left(\tilde{\omega} \hat{p}_{n}\right)\right)^{-1}\left[\hat{p}^{\text {trial }}-\kappa \tilde{\gamma}_{n+1} \frac{\partial \phi_{p}\left(\hat{p}_{n}\right)}{\partial p}\right]
\end{aligned}
$$

In Box (2), the complete algorithm for the Impl-Ex method can be found.

\subsection{Momentum balance equation and the effective tangent modulus}

The Principle of the Virtual Power, applied to the body $\Omega$ at step $(n+1)$, is verified by using the explicit stresses, as follows:

$$
\int_{\Omega}\left(\tilde{\boldsymbol{\sigma}}_{n+1}: \nabla^{s} \delta \boldsymbol{u}\right) \mathrm{d} \Omega-\int_{\Omega}\left(\boldsymbol{f}^{\text {ext }} \cdot \delta \boldsymbol{u}\right) \mathrm{d} \Omega=0 ; \quad \text { for all admissible } \delta \boldsymbol{u}
$$

In the context of the finite element method, Eq. (48) is discretized and generally solved by means of a Newton-Raphson scheme. In that case, given the residue of the internal and external forces:

$$
\boldsymbol{R e s}=\int_{\Omega}\left(\tilde{\boldsymbol{\sigma}}_{n+1}: \nabla^{s} \delta \boldsymbol{u}\right) \mathrm{d} \Omega-\int_{\Omega}\left(\boldsymbol{f}^{\text {ext }} \cdot \delta \boldsymbol{u}\right) \mathrm{d} \Omega
$$

the discrete body incremental displacement, $\Delta \mathbf{u}$, are found via an iterative procedure, where every iteration solves:

$$
K \Delta \mathbf{u}=-\operatorname{Res}
$$

The stiffness matrix $\boldsymbol{K}=\partial \mathbf{R e s} / \partial \mathbf{u}$ results from the assembling of the elemental stiffness matrices $\boldsymbol{K}^{\mathrm{e}}$ as follows:

Box 2: Impl-Ex numerical integration algorithm

$$
\text { Integration step: }(\mathrm{n}+1)
$$

INPUT: $\boldsymbol{\varepsilon}_{n+1}$; implicit previous values: $\hat{\boldsymbol{X}}_{n}, \hat{p}_{n}, \hat{\gamma}_{n}$

(1) Implicit integration

CALL IMPLICIT ROUTINE (Box (1))

OUTPUT: $\hat{\boldsymbol{\sigma}}_{n+1} ; \hat{\boldsymbol{X}}_{n+1} ; \hat{p}^{\text {trial }} ; \hat{\boldsymbol{S}}^{\text {trial }}$

(2) Internal variables extrapolations from previous implicit values

$$
\begin{aligned}
& \tilde{\gamma}_{n+1}=\hat{\gamma}_{n} \frac{\Delta t_{n+1}}{\Delta t_{n}} \\
& \tilde{\bar{\varepsilon}}_{n+1}=\hat{\bar{\varepsilon}}_{n} \\
& \tilde{f}_{n+1}=\hat{f}_{n}
\end{aligned}
$$


(3) Update stress state: $\tilde{\boldsymbol{\sigma}}_{n+1}$

Evaluate: $\beta_{1}=1+\left[6 \mu \tilde{\gamma}_{n+1} / \tilde{\sigma}_{0 n+1}^{2}\right]$

Evaluate: $\beta_{2}=1+\kappa \tilde{\gamma}_{n+1} \tilde{\chi} \tilde{\omega}^{2} \cosh \left(\tilde{\omega} \hat{p}_{n}\right)$

where: $\tilde{\chi}=2 q_{1} \tilde{f}_{n+1}^{*} ; \tilde{\omega}=-\frac{3}{2} \frac{q_{2}}{\tilde{\sigma}_{0 n+1}^{2}}$

Compute : $\widetilde{\boldsymbol{S}}_{n+1}=\frac{1}{\beta_{1}} \widehat{\boldsymbol{S}}^{\text {trial }}$

$$
\begin{aligned}
& \tilde{p}_{n+1}=\frac{1}{\beta_{2}}\left[\hat{p}^{\text {trial }}-\kappa \tilde{\gamma}_{n+1} \frac{\partial \phi_{p}\left(\hat{p}_{n}\right)}{\partial p}\right] \\
& \tilde{\boldsymbol{\sigma}}_{n+1}=-\tilde{p}_{n+1} 1+\tilde{\boldsymbol{S}}_{n+1}
\end{aligned}
$$

OUTPUT: explicit values: $\tilde{\boldsymbol{\sigma}}_{n+1}, \widetilde{\boldsymbol{X}}_{n+1} ;$ implicit values : $\hat{p}_{n+1}, \widehat{\boldsymbol{X}}_{n+1}$

$$
\boldsymbol{K}=\sum_{e=1}^{\text {nelem }}\left(\Lambda^{\mathrm{e}} \boldsymbol{K}^{e}\right) ; \quad \boldsymbol{K}^{e}=\int_{\Omega^{\mathrm{e}}}\left(\boldsymbol{B}^{T} \boldsymbol{C}_{n+1}^{\mathrm{eff}} \boldsymbol{B}\right) \mathrm{d} \Omega
$$

where $\Lambda^{\mathrm{e}}$ is the standard assembling operator, $\boldsymbol{B}$ is the elemental strain-displacement matrix and $\boldsymbol{C}_{n+1}^{\mathrm{eff}}$ the effective tangent tensor:

$$
\boldsymbol{C}_{n+1}^{\mathrm{eff}}=\frac{\partial \tilde{\boldsymbol{\sigma}}_{n+1}}{\partial \boldsymbol{\varepsilon}_{n+1}}
$$

The fundamental property that we have preserved with Eqs. (43)-(47), is that $\psi$ results a quadratic and convex function on the variables $\tilde{p}$ and $\tilde{q}$, implying that the Hessian matrix $\boldsymbol{H}=\partial^{2} \psi / \partial \boldsymbol{\sigma}^{2}$ will be a positive definite tensor. This important aspect defines an effective tangent tensor, $\boldsymbol{C}_{n+1}^{\mathrm{eff}}$, that is always a positive definite and constant (not depending of $\left.\Delta \boldsymbol{\varepsilon}_{n+1}\right)$ term, even when material failure is present during the numerical simulation process. From Eqs. (43)-(46), $C_{n+1}^{\text {eff }}$ is given by

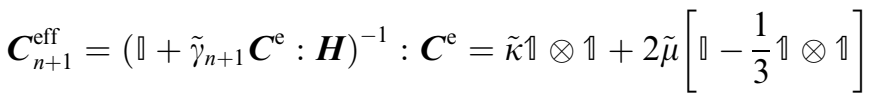

$$
\begin{aligned}
& \tilde{\mu}=\mu\left[1+\frac{6 \mu \tilde{\gamma}_{n+1}}{\tilde{\sigma}_{0 n+1}^{2}}\right]^{-1} ; \quad \tilde{\mu}>0 . \\
& \tilde{\kappa}=\kappa\left(1+\kappa \tilde{\gamma}_{n+1} \tilde{\chi} \tilde{\omega}^{2} \cosh \left(\tilde{\omega} \hat{p}_{n}\right)\right)^{-1} ; \quad \tilde{\kappa}>0,
\end{aligned}
$$

where the fourth and second order identity tensors, $\square$ and 1, are defined in Appendix A.2.

It is then straightforward to show that the positive definite character of $\boldsymbol{C}_{n+1}^{\mathrm{eff}}$ is inherited by the finite element global stiffness matrix (51) determining a numerically well-posed equation set (50). Furthermore, due to the constant character of the $\boldsymbol{C}^{\mathrm{eff}}$ tensor, the global equation system solution (48) requires only one iteration per time step to converge. Both properties characterize the high performance of the Impl-Ex algorithm when compared with the implicit method of Section 4.1.

In the next section we study, by means of a typical example, the convergence rate of the Impl-Ex procedure compared with the implicit version. Also the comparative computational cost in every case, is presented. 


\section{The tensile bar test}

The GTN material model has the feature of capturing dissimilar localization modes, which, in general, correspond with different mechanisms inducing the material failure. They are: (a) shear bands, where predominant deviatoric strains are developed into the strain localized zone, (b) mode I of fracture, where dominant spherical strains are observed in the same zone. The last failure mode is typically observed in quasi-brittle crack growth problems.

This constitutive model property is analyzed in the present section by means of the tensile bar test. We model it until reaching the total loss of the structural load carrying capacity. A rectangular bar in plane strain and a round bar subjected to axial symmetry conditions are simulated. Even when these solutions have been presented in the 80's by Needleman and Tvergaard; in the following subsections we remark two aspects of this problem:

(i) the role played by the bifurcation condition $\left(\operatorname{det}\left[\boldsymbol{Q}^{\mathrm{ep}}\right]=0\right)$ and the angle $\theta$, solution of the equation $\operatorname{det}\left[\boldsymbol{Q}^{\mathrm{ep}}(\boldsymbol{\eta}(\theta))\right]=0$, in the transition from a shear band to an opening localization mode. Such mechanism is observed where a high stress triaxiality is developed.

(ii) the algorithmic issues related to the finite element approach: the pathological dependence of the numerical results with the mesh size and the integration scheme accuracy.

The main features of the failure mechanisms for both bars, with material data corresponding to a steel, can be observed in Fig. 4. The results of that figure correspond to the end of analysis, when the complete loss of the bar load carrying capacity has been reached. Fig. 4a shows in black the finite elements that remain in the plastic loading condition. This figure, jointly with Fig. 4d, displays clearly the strain localization and deformation modes related to each bar type: a shear band formation for plane strain condition and an opening mode I for axial symmetry condition. The zones displaying a void fraction $f>0.2$, colored in dark gray in Fig. 4c, could be interpreted as that material reaching the complete stress exhaustion. The round bar plastic strain in Fig. 4e shows the trend to form the characteristic cup-cone fracture mode observed in this test. Additional details about this numerical simulation aspect (the cup-cone formation) have been presented in the work of Besson et al. (2003). The necking observed in the round bar example is the result of a global bifurcation mode as a consequence of the material instability. In both test, the onset of localization process, in the central part of the bar, is induced by means of a small geometrical imperfection.

\subsection{Localization and analytical bifurcation modes in the round bar test}

It is a well known fact that the analytical bifurcation analysis of the Section 3.2, based on determining the first time $t_{B}$ that Eq. (24) is verified for the critical angle $\theta^{\text {crit }}$ given by Eq. (26), does not necessarily determine neither the global mode of localization nor the onset time of it. Therefore, the strain localization response, understood as the process inducing plastic loading in a thin material slice while the neighborhood (bulk) material is elastically unloading, is intrinsically related with a material instability phenomenon, 


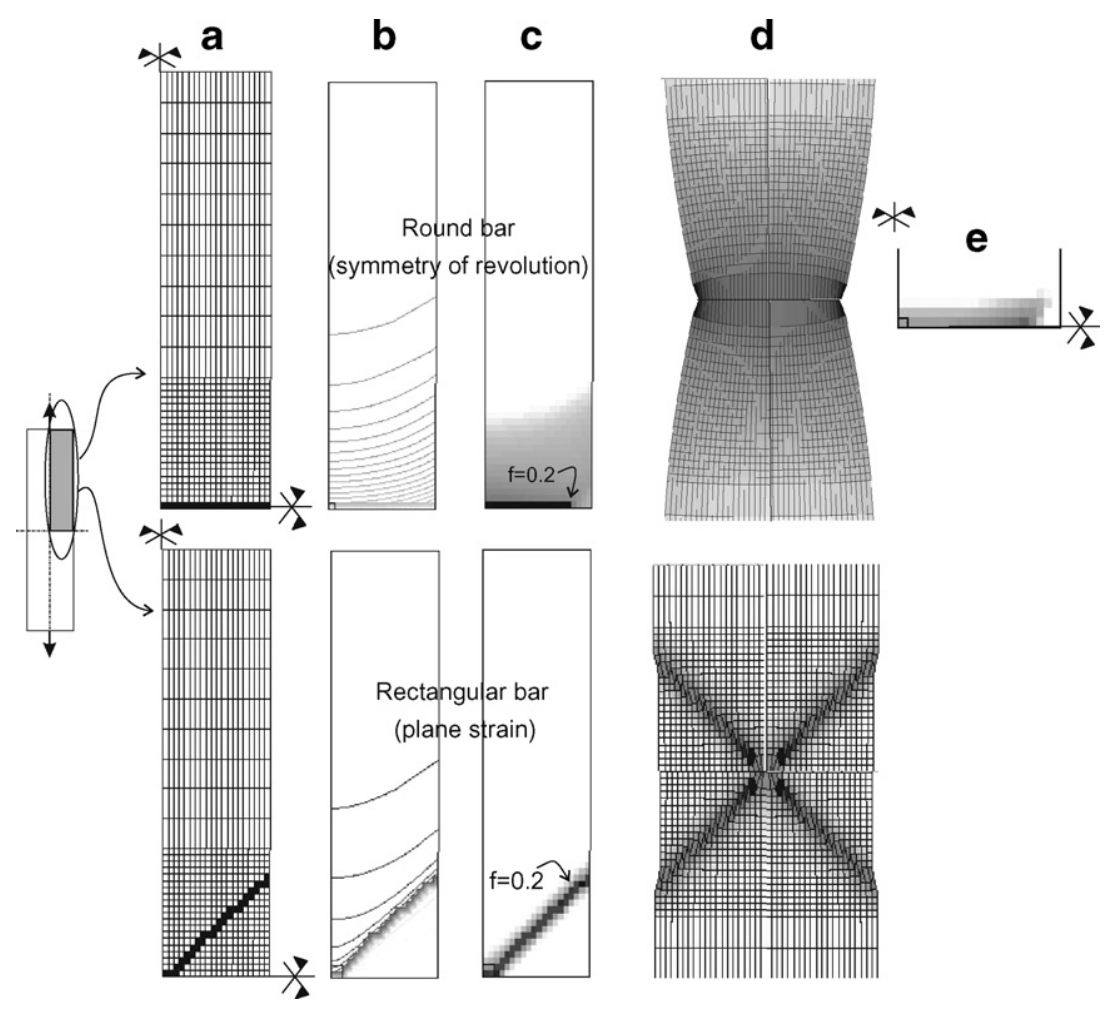

Fig. 4. Predicted strain localization modes for a round (symmetry of revolution) and a rectangular (plane strain) bar. BBAR finite element meshes with aspect ratio (in the necking zone) equal to 1 have been used: (a) Finite elements verifying loading state in the final stages of the analysis. (b) Contour lines of iso-displacements. (c) Void fraction contour fill " $f$ ". (d) Finite element mesh in the deformed configuration. (e) Microscopic equivalent plastic strain map " $\bar{\varepsilon} "$.

Rice (1976). Therefore, it is necessary that the material instability condition (24) should be verified for the observed localization angle $\left(\theta^{\text {loc }}\right)$ in correspondence with the direction of the strain localized band, and time $t^{\text {loc }}>t_{B}$, even when they are not coincident with the results of Eq. (26) at time $t_{B}$.

In the present section we analyze the relation between those concepts in the round bar tensile test. Performing the analytical bifurcation analysis of the preceding section, Eq. (26) gives the critical angle $\theta^{\text {crit }}=35^{\circ}$ when Eq. (24) is first verified at the bar central zone $\left(t=t_{B}\right)$. It means that the possible localization band would form an angle of $35^{\circ}$ with the horizontal axis. Fig. $5 \mathrm{~b}$ plots the distribution of the orthogonal directions to $\eta^{\text {crit }}$ defined by the angle $\theta^{\text {crit }}$ on the complete bar (even when the central point is the only one verifying $\operatorname{det}\left[Q^{\mathrm{ep}}\right]=0$ at that instant). Furthermore, Fig. 5c displays in light gray, and at the same time, those finite elements which are in a plastic loading condition. Clearly, it could be observed that this time does not correspond with the beginning of the strain localization process.

After an additional period of time, at $t^{\text {loc }}>t_{B}$, the strain localization process begins at the bar center. The thin material slice in plastic loading follows an horizontal direction, see Fig. 6b. Theoretical angle determined by Eq. (26), providing the minimum of $\operatorname{det}\left[\boldsymbol{Q}^{\mathrm{ep}}\right]$, remains to be approximately $\theta^{\text {crit }} \approx 35^{\circ}$ with $\operatorname{det}\left[\boldsymbol{Q}^{\text {ep }}\left(H, \boldsymbol{\eta}^{\text {crit }}\left(\theta^{\text {crit }}\right), t^{\text {loc }}\right)\right]<0$, see Fig. 6a. 
a

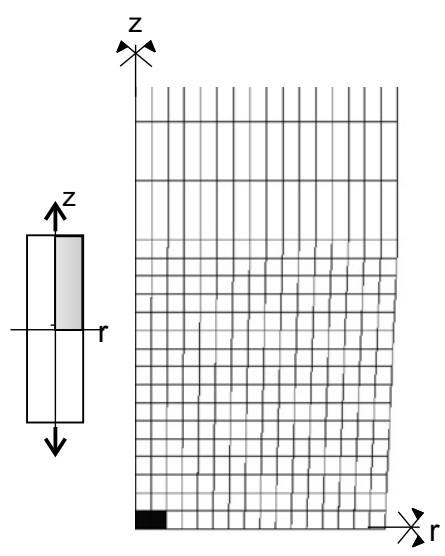

b

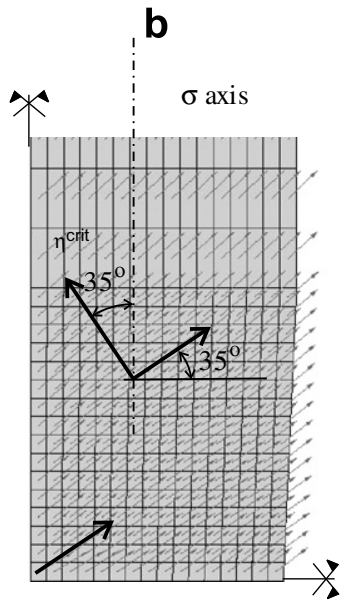

C

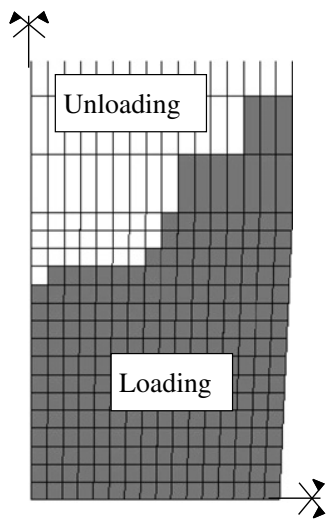

Fig. 5. Analytical solution of instability problem applied to the axi-symmetric bar test: (a) First finite elements that fulfill the critical material instability condition (Eq. (27)). (b) Critical bifurcation direction $\eta^{\text {crit }}$, computed from Eq. (31). (c) Loading-unloading state. All figures correspond to the same integration time $\operatorname{step}\left(t=t_{B}\right)$.

However, in the same instant, the term $\operatorname{det}\left[\boldsymbol{Q}^{\mathrm{ep}}\left(H, \boldsymbol{\eta}\left(\theta^{\mathrm{loc}}\right), t^{\mathrm{loc}}\right)\right]=0$ for an angle $\theta^{\mathrm{loc}} \approx 0^{\circ}$. This situation means that a material instability condition holds for approximately the horizontal direction, in correspondence with the strain localization pattern observed. In the upper part of Fig. $6 \mathrm{~b}$ we show, in black, the finite elements that verify the condition:

$$
\operatorname{det}\left[\boldsymbol{Q}^{\mathrm{ep}}\left(H, \boldsymbol{\eta}\left(\theta^{\text {loc }}\right), t^{\text {loc }}\right)\right]=0 ; \quad \text { for } \theta^{\text {loc }} \approx 0^{\circ}
$$

In subsequent times $\left(t_{2}>t^{\text {loc }}\right.$ and $\left.t_{3}>t_{2}\right)$, Fig. $6 \mathrm{c}$ and $\mathrm{d}$ show similar results. It can be observed the close correspondence between the condition (56) and the evolution of the strain localization pattern that is developing in the center of the bar. We conclude that the "initial" critical direction, determined by the first time that the acoustic tensor becomes singular, is not compatible with the expected cup-cone localization mode, and therefore, the localization process does not begin at that time. However, at a subsequent time, it is observed a different direction, making singular the acoustic tensor that produces strain localization. This direction agrees with the typical cup-cone failure mode geometry.

This response differs significantly from the rectangular bar test solution, where a shear band localizes with an angle $\theta^{\text {crit }} \approx 45^{\circ}$ coinciding very approximately with the solution of problem (26), (27) and generating a kinematically admissible mechanism of deformation.

\subsection{Mesh dependence analysis of the round bar tensile test}

A well know consequence of adopting a constitutive model not having a characteristic length, like the GTN one, is that the material instability induces a pathological dependence of the numerical results with the mesh size. In this subsection, we analyze this spurious response by means of the above presented round bar tensile test.

Three uniform meshes (M1, M2 and M3) of BBAR finite elements having aspect ratios equal to 1 are used, see Fig. 7a:c. The material parameters, corresponding to a typical 


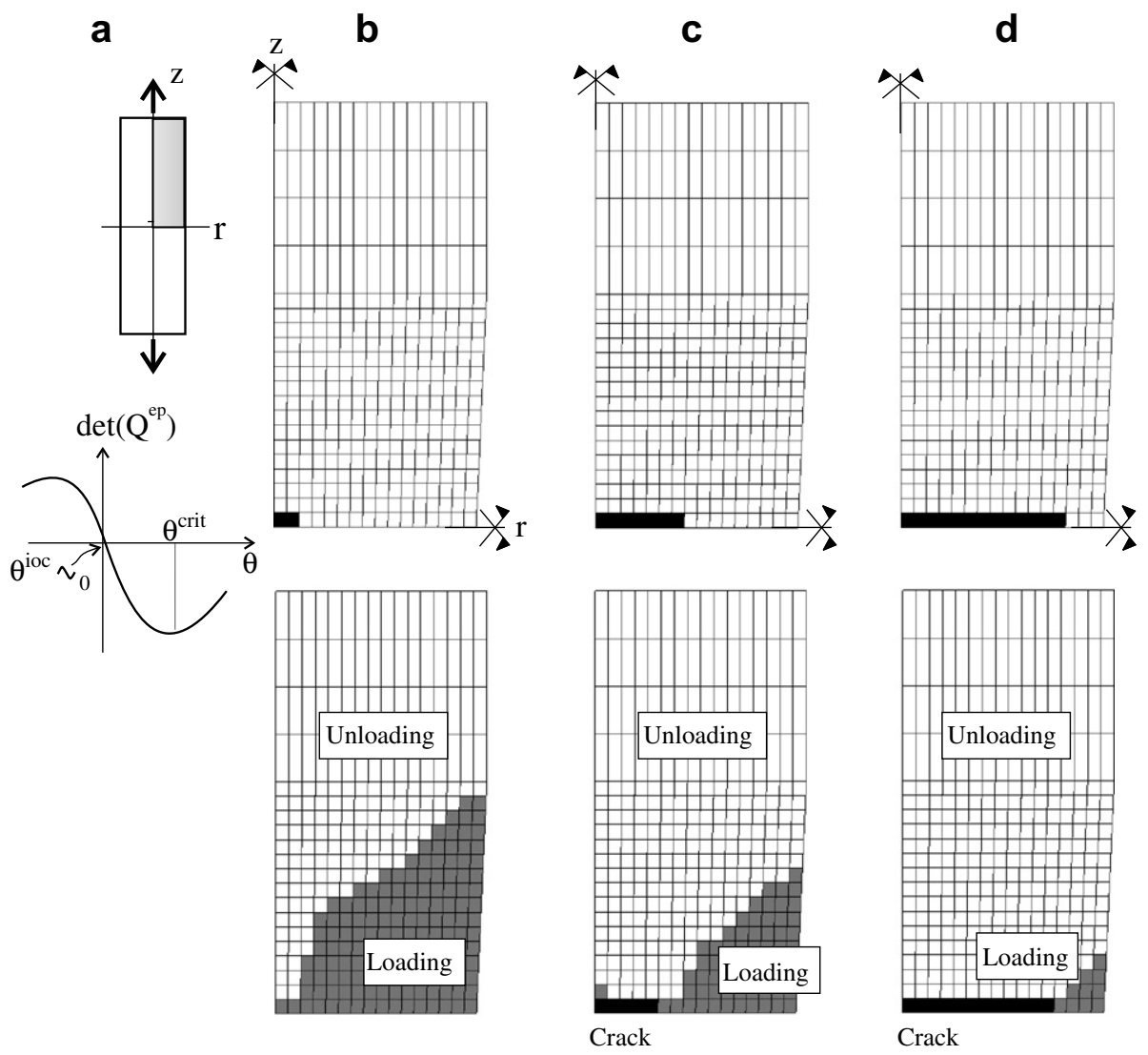

Fig. 6. Failure process induced by ductile fracture in mode I. (a) Differences between the angles $\theta^{\text {crit }}$ and $\theta^{\text {loc }}$. (b)-(d) Comparison between the patch of finite elements satisfying the material bifurcation condition for $\theta^{\text {loc }} \approx 0$ (up) and the patch of finite elements in loading-unloading regime (down) describing the strain localization process evolution. Both figures, in each column, correspond to identical integration time steps $\left(t_{1}, t_{2}\right.$ and $t_{3}$ respectively).

a

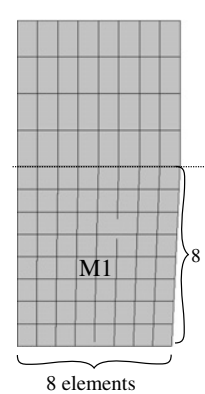

b

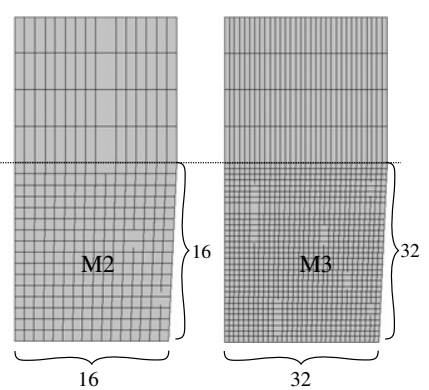

d

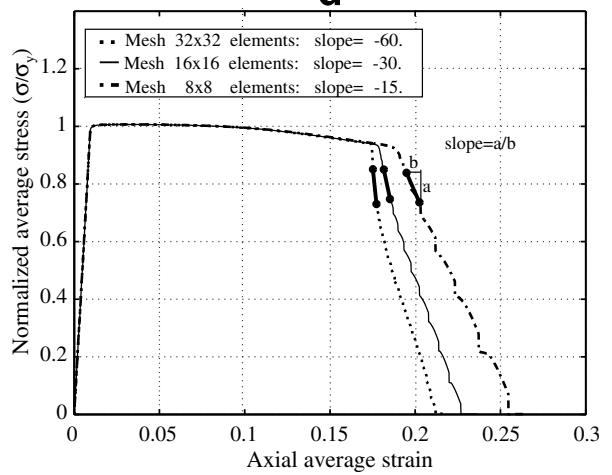

Fig. 7. Mesh size sensitivity analysis: (a)-(c) BBAR finite element meshes "M1, M2 and M3". (d) Stress vs. axial strain curves for the three used discretization levels (M1, M2 and M3). 
steel, are: $q_{1}=1.5, q_{2}=1.0, q_{3}=2.25$, initial void fraction $f_{0}=0.01, f_{\mathrm{N}}=0.04, s_{\mathrm{N}}=0.1$, $\varepsilon_{\mathrm{N}}=0.3, \sigma_{\mathrm{y}} / E=1 / 100, v=0.3, f_{\mathrm{C}}=0.15, f_{\mathrm{F}}=0.25$. We assume a material without hardening $(H=0)$.

Fig. 7d plots the load (nominal average stress) vs. displacement (average strain) curves for the three meshes. The complete loss of structural load carrying capacity is obtained by removing those finite elements where some of their Gauss Point have reached a void fraction level $f>0.2$. We have not detected a substantial change of the structural response by choosing a higher value of $f$ for removing them. This technique has been widely used in ductile fracture simulation, see Tvergaard and Needleman (2006).

From Fig. 7d and after the localization process begins, we can observe the strong dependence of the post-critical slope, and therefore the dependence of the dissipated structural energy, with the mesh size, in this stage.

Clearly, a constitutive model regularization becomes mandatory in this type of problems.

\subsection{Convergence and cost analysis of the integration schema}

An additional topic studied by means of the same round bar tensile test is the convergence rate of the implicit and Impl-Ex numerical integration algorithms.

Adopting the same material model of the previous subsection, we obtain several solutions with the most refined mesh (M3) using both algorithms, the implicit and the ImplEx. Every run has a fixed integration time step length along the complete analysis. These solutions are plotted in Fig. 8a. The numerical simulations were controlled by imposing an incremental top displacement in the specimen, being that the time step length $\Delta t^{*}$ corresponds to an incremental average strain of value: $\Delta \varepsilon=2.3 \times 10^{-4}$.

The convergence rates of both integration algorithms are plotted in Fig. 8b. We adopt the implicit solution with $\Delta t^{*} / 8$ as the exact one, and compute the norm- $L_{2}$ of the error corresponding to the other numerical solutions as follows:

$$
\|e\|_{L_{2}}=\left(\int\left(\Sigma-\Sigma_{\mathrm{ex}}\right)^{2} \mathrm{~d} \varepsilon\right)^{1 / 2}
$$
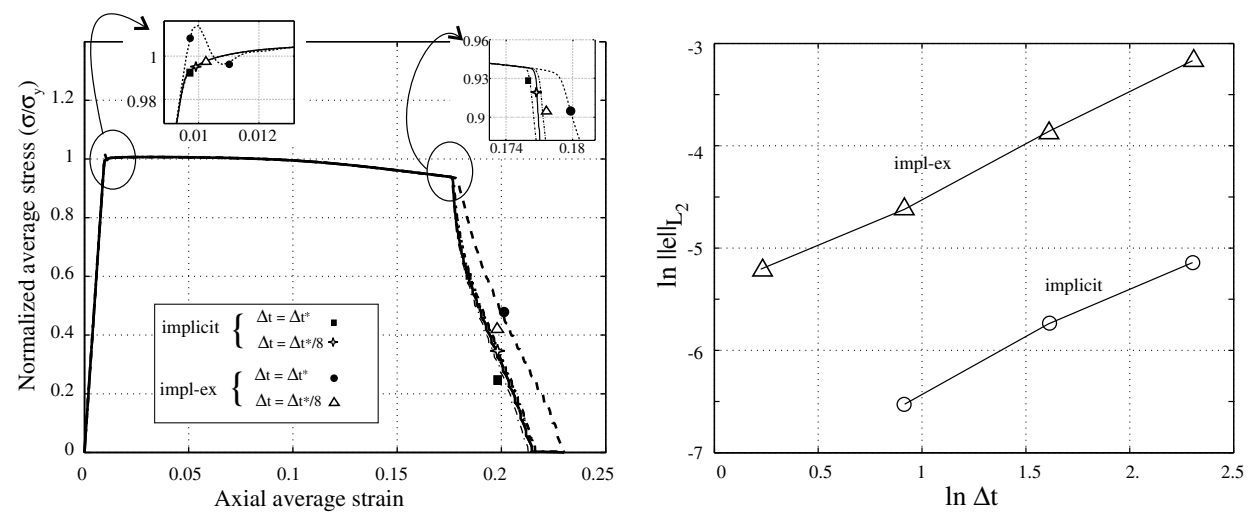

Fig. 8. Convergence rate analysis. 
Table 1

Relative computational cost analysis: Implicit vs. Impl-Ex algorithms

\begin{tabular}{llll}
\hline Integration method & Step length & Mean number of iterations & Relative CPU time \\
\hline Implicit & $\Delta t^{*}$ & $4-5$ & 1 \\
tol.: $1 . \times 10^{-3}$ & & $(3-4)$ & $(0.67)$ \\
$\left(\right.$ tol.: $\left.1 . \times 10^{-1}\right)$ & $\Delta t^{*} / 2$ & $4-6$ & 1.9 \\
& & $(2-3)$ & $(1.1)$ \\
Impl-Ex & $\Delta t^{*} / 4$ & $4-6$ & 3.4 \\
& & $(2-3)$ & $(1.9)$ \\
& $\Delta t^{*}$ & 1 & 0.15 \\
& $\Delta t^{*} / 2$ & 1 & 0.30 \\
& $\Delta t^{*} / 4$ & 1 & 0.60 \\
& $\Delta t^{*} / 8$ & 1 & 1.2 \\
\hline
\end{tabular}

where $\Sigma$ is the normalized average stress load/area $\left(\Sigma_{\text {ex }}\right.$ is the normalized average stress for the assumed exact solution) and $\varepsilon$ the axial mean strain. Although the error of the implicit procedure is smaller than the Impl-Ex one using identical time step increments, both solutions present identical convergence rates.

Table 1 provides a comparative analysis of the computational cost demanded by every solution. The implicit solutions requires more CPU time as the tolerance of the equilibrium equation is more adjusted. The CPU time cost of the Impl-Ex scheme, using $\Delta t^{*} /$ 8 , is 1.2 times larger than the implicit strategy cost with $\Delta t^{*}$.

Respect to the comparative analysis between both algorithms, an important additional remark should be mentioned. As the loading-unloading process arises in the center of the bar, the implicit algorithm displays a dramatic loss of robustness, and therefore, the loss of convergence becomes a very serious drawback. Alternatively, due to the effective tangent tensor properties ( $\boldsymbol{C}^{\text {eff }}$, from Eq. (53)), the Impl-Ex procedure provides a global stiffness matrix that remains positive definite and constant throughout the complete analysis. As a direct consequence, the numerical solution is well-posed in every time step and requires only one iteration to converge. This feature provides a notable improvement of the algorithmic robustness.

\section{Ductile fracture analysis in plane strain}

In a recent work, Tvergaard and Needleman (2006) simulate the crack growth resistance of a structural steel assuming that the material has two well separated scales of void sizes, of the order 1 and $100 \mu \mathrm{m}$. The growth and coalescence of the larger voids are explicitly modeled by means of islands of softer material. They are embedded in a matrix material with a smaller scale of void distribution. The smaller scale void fraction evolution (growth and coalescence) is described by a GTN constitutive material model.

Those authors perform a full 3D analysis by considering a slice of material. The larger void distribution are represented by spherical islands embedded in the matrix, and the matrix is subjected to boundary conditions resembling a plane strain state. Their results are compared with a typical $2 \mathrm{D}$ analysis that the same authors had published previously.

In this section, we simulate a similar problem by means of a plane strain 2D model, The larger void size are assumed to be hollow cylinders. Our results are compared with the plane strain ones of Tvergaard and Needleman (2006). 
Two aspects are mainly addressed in the present study:

(i) In the first part, we analyze the dependence of the results with the mesh size. A posterior discussion is presented.

(ii) A second analysis is addressed to understand the relation between the material stability condition with the observed strain localization mode.

\subsection{Numerical model}

A plane strain slice of a material having two scales of void sizes is studied. The domain of analysis corresponds to an enough large crack tip neighborhood to verify the small scale yielding assumption. Displacement boundary conditions are imposed on the boundary $A-B-C$, see Fig. 9b, and given by

$$
\begin{aligned}
& u_{x}=\frac{2(1+v) K_{\mathrm{I}}}{E} \sqrt{\frac{r}{2 \pi}} \cos (\theta / 2)\left[1-2 v+\sin ^{2}(\theta / 2)\right] \\
& u_{\mathrm{y}}=\frac{2(1+v) K_{\mathrm{I}}}{E} \sqrt{\frac{r}{2 \pi}} \sin (\theta / 2)\left[2-2 v-\cos ^{2}(\theta / 2)\right]
\end{aligned}
$$

This displacement distribution is compatible with the singular stress field of the linear elastic solution, the $K$-field, around a sharp crack loaded in mode I. This classical solution could be found in standard textbooks on Fracture Mechanics. The stress intensity factor $K_{\mathrm{I}}$ is related with the integral $J$ through: $J=\frac{K_{\mathrm{I}}^{2}\left(1-v^{2}\right)}{E}$. In Eqs. (58), (59), $r$ and $\theta$ are the polar coordinates of the point.
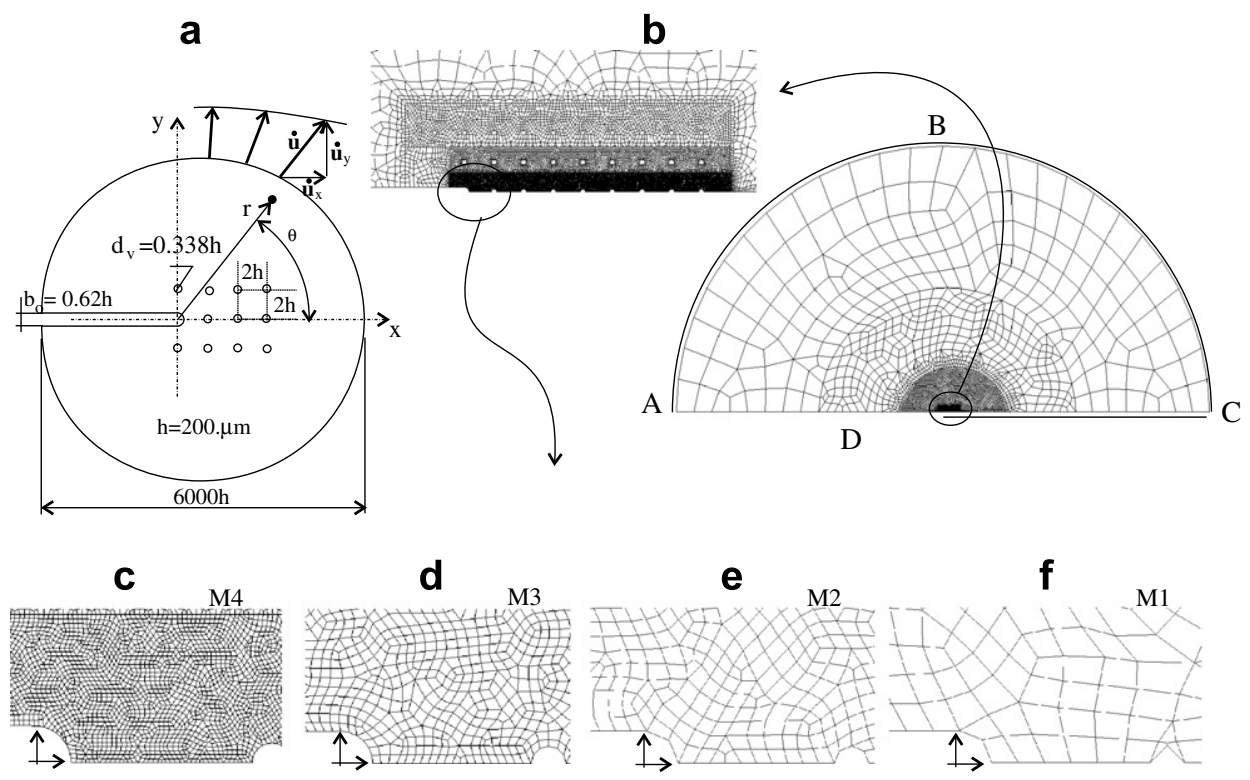

Fig. 9. Ductile crack growth. Finite element model: Meshes M1 (element size $\approx 60 \mu \mathrm{m}$ ), M2 (element size $\approx 30 \mu \mathrm{m})$, M3 (element size $\approx 15 \mu \mathrm{m})$, M4 (element size $\approx 8 \mu \mathrm{m})$. 
In the same Fig. 9a, it can be seen the pattern that we have adopted for the cylindrical void distribution. The reference length size is $h=200 \mu \mathrm{m}$ and the cylindrical void diameters are: $d_{v}=0.338 h$ what is equivalent (for the larger void scale) to a volume void fraction $f=0.045$.

The material is characterized by the following parameters: $\sigma_{\mathrm{y}} / E=0.0025$, Poisson's ratio $v=0.3$, initial void fraction $f_{0}=0.0005, f_{\mathrm{N}}=0.04, s_{\mathrm{N}}=0.1$ and $\varepsilon_{\mathrm{N}}=0.3$. The material hardening is described by the following law:

$$
\frac{\sigma_{0}}{\sigma_{\mathrm{y}}}=\left[1+\frac{E \bar{\varepsilon}}{\sigma_{\mathrm{y}}}\right]^{N}
$$

where $N=0.1$.

\subsection{Sensitivity analysis of the results with the mesh size}

We solve the problem by using four BBAR finite element quadrilateral meshes: $M 1, M 2, M 3$ and $M 4$, see Fig. 9c and f. The element sizes in that meshes range from the order of 1 to 0.1 times the radius of the cylindrical larger voids.

In Fig. 10a we plot, for the four meshes, the curves corresponding to the crack mouth opening displacement $\left(b-b_{o}\right)$ (scaled by the size $\left.b_{o}=0.62 h\right)$ vs. the load level. The last term, the load level, is characterized by the scaled integral $J\left(J /\left(\sigma_{o} b_{o}\right)\right)$. These results are compared with the solution presented in Tvergaard and Needleman (2006). Fig. 10b displays the crack growth resistance curves for all meshes and their comparison with the reference work.

The first conclusion we can obtain from this comparison is the following one. Even when both models show some important differences between them, such as (a) a linear kinematics vs. the exact one in Tvergaard and Needleman (2006); or (b) an inviscid material law vs. the viscous one of the same reference; there are not significant differences between both results. Much more important differences arise when comparing the 3D analysis results assuming spherical voids respect to the 2D plane strain model results with cylindrical voids.
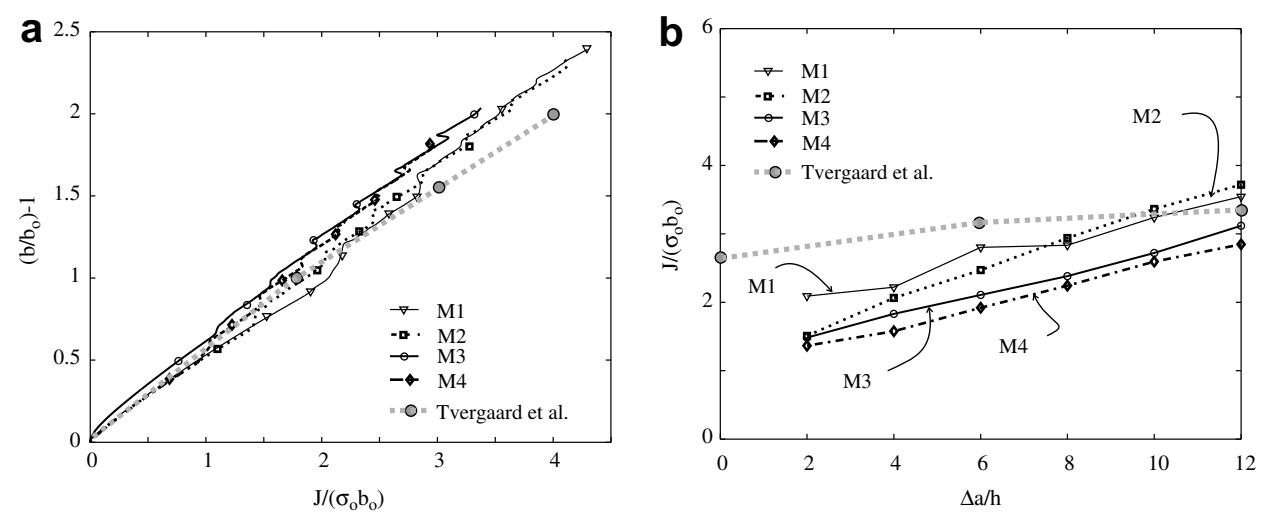

Fig. 10. Ductile crack growth. Result dependence with the size mesh: (a) Crack mouth opening vs. $J$ curves. (b) crack growth resistance curves. 
An additional important remark can be reported. It was mentioned in the literature that, in the crack growth problem, the voids of larger size determine a characteristic length that implicitly induces a constitutive model regularization and yields a numerical response insensibles to the mesh size. However, this conclusion is not clear for us. Fig. 10b does not show a response being clearly mesh size independent. In this aspect, we consider that we need to perform further studies addressed to determine the relative weight of the dissipated energy into the strain localization zone $\left(\Gamma_{0}\right)$, that can be considered as the energy spent in the creation of a new crack surface, respect to the total dissipated energy of the loading process $(\Gamma)$. It should be considered that an important part of $(\Gamma)$ corresponds to the stable plastic deformation dissipated energy $\left(\Gamma_{p}\right)$ close to the crack tip. We know that the first component $\left(\Gamma_{0}\right)$ of the total energy $(\Gamma)$ is strongly dependent of the mesh size, tending to zero as the mesh size goes to zero, which is not an admissible physical behavior. We mention the contributions of Tvergaard and Hutchinson (1992) and Pardoen et al. (2004) who have analyzed the relative weight between those energies.

\subsection{Analytical bifurcation condition vs. strain localization modes}

In Fig. 11a the void fraction, at the end of the analysis, is displayed. This result is obtained by removing those finite elements having some Gauss Point with a void fraction greater than 0.2. In the same figure, the removed finite elements are painted with dark gray. It could be observed in the most fine mesh that the crack growth follows an irregular horizontal line $(x-y$ plane). The damage begins in a large void island trying to capture a shear band mode at $45^{\circ}$, see the plastic strain pattern in Fig. 11b. However, the high stress triaxiality existing along the horizontal direction, between two contiguous voids, makes the volume void fraction (for the small void scale) grows faster, forcing the coalescence of two contiguous horizontal large voids and inducing the material exhaustion in that direction.

In Fig. 11c and e we analyze, in a sequence of time steps $(240, \ldots, 260)$, the relation between the analytical bifurcation condition, given by Eq. (26), (27), and the strain localization mode above described. The sequence of figures in column "e" shows the material zone where the instability condition $\operatorname{det}\left[\boldsymbol{Q}^{\mathrm{ep}}\right]=0$ is detected. Initially, at the time step 240, it is observed that the material stability is lost in a large zone, close to the crack tip. In the zone between two horizontal contiguous large voids, the critical angle which makes minimum the $\operatorname{det}\left[Q^{\text {ep }}\right]$, ranges from $\theta^{\text {crit }} \approx 30^{\circ}$ to $40^{\circ}$ respect to the vertical line. In that stage, neither the direction of the critical angle coincides with the strain localization pattern finally developed, nor the typical plastic loading/unloading process (across a bandwidth size of only one element) starts to happens, see the sequence of pictures in Fig. 11d. It is not until the step 248 that the loading/unloading process in point $\mathrm{P}$ begins. It could be observed in the same instant (column "e") that the condition $\operatorname{det}\left[\boldsymbol{Q}^{\mathrm{ep}}\right]=0$ is obtained for an angle $\theta^{\text {loc }} \approx 0^{\circ}$. Furthermore, in the same instant, column "c" shows that $f<f_{c}$ at the $\mathrm{P}$ point. It is only after the step 250 that the material reach a void fraction $f>f_{c}$. Clearly, in this case, $f_{c}$ is not the value that induces localization.

A similar conclusion like that in Section 5.1 is then obtained. The "initial" critical direction $\left(\theta^{\text {crit }}\right)$ compatible with the first time $\left(t_{B}\right)$ that the acoustic tensor becomes singular, does not necessarily induce the localization process (loading/unloading mechanism). However, at a subsequent time $\left(t^{\text {loc }}\right)$, it is observed a different angular direction $\left(\theta^{\text {loc }}\right)$, very close to zero, producing a strain localization mechanism compatible with a crack propagation opening in mode I. 
a

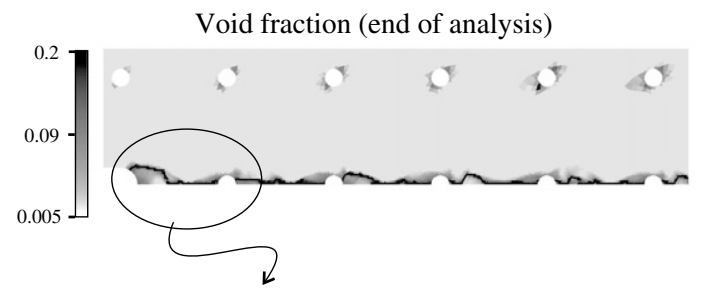

b

Plastic strain (step 246)

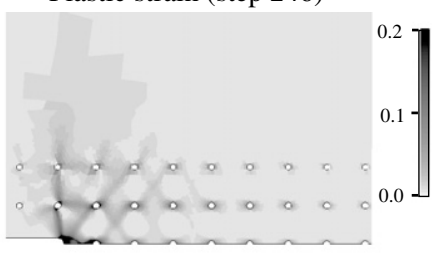

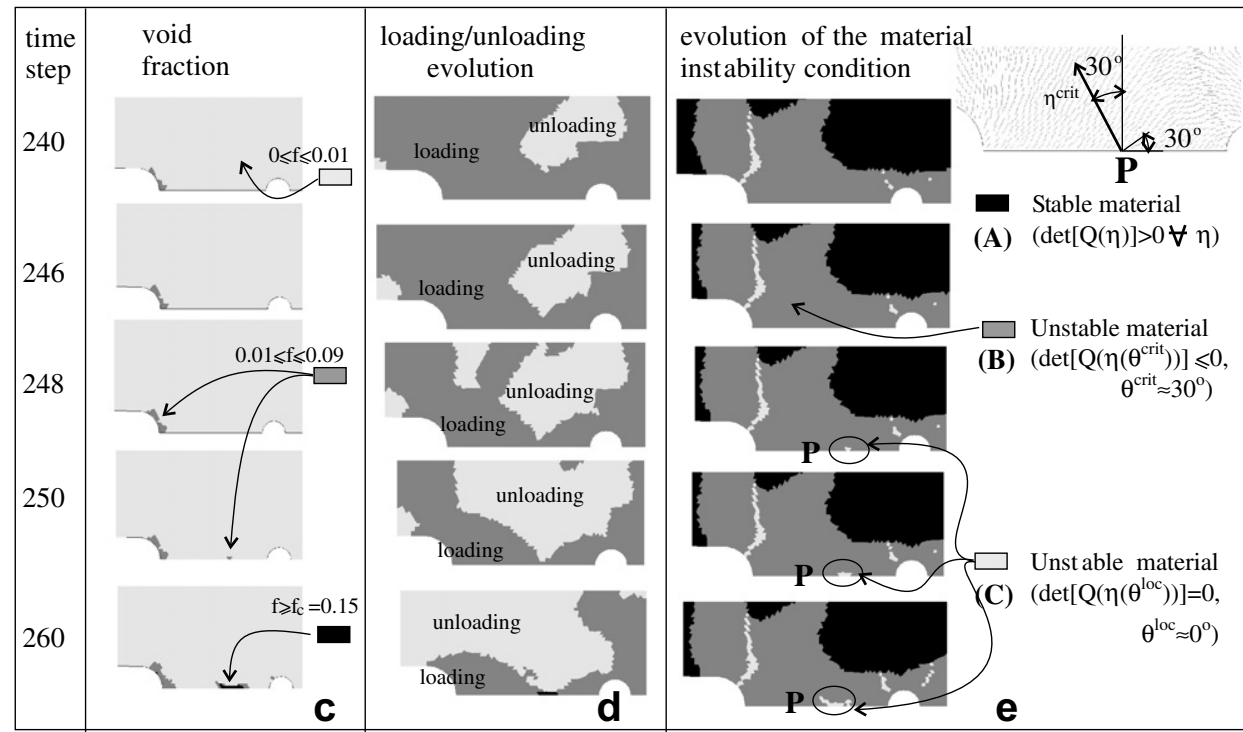

Fig. 11. Ductile crack growth. Results of the void-coalescence phenomenon.

\section{Conclusions}

Several topics addressed to the numerical solution of ductile fracture problems by means of numerical methods, using the GTN continuum constitutive model, have been presented:

(i) An analytical strategy to determine the material critical bifurcation condition in closed form for the most general 3D cases. With this procedure we have analyzed the critical angle that makes minimum the determinant of the acoustic tensor. It has been remarked that, under high stress triaxiality effects, a critical angle close to zero, respect to the maximum principal stress, is admissible. In those cases, where localization happens just when the critical condition is verified (with $\theta^{\text {crit }} \approx 0^{\circ}$ ), this property could induces strain localization mode I of fracture. Alternatively, being the critical angle $\theta^{\text {crit }} \neq 0$ at $t_{B}$, a mode $\mathrm{I}$ of failure has been observed when the strain localization process is delayed in such a way that at a posterior time $t^{\text {loc }}>t_{B}$, the angle $\theta^{\text {loc }} \approx 0$ fulfills the material instability condition $\left(\operatorname{det}\left[\boldsymbol{Q}^{\mathrm{ep}}\left(\boldsymbol{\eta}^{\mathrm{loc}}\left(\theta^{\mathrm{loc}}\right)\right)\right]=0\right)$.

(ii) The Impl-Ex integration algorithm has become a fundamental numerical tool for obtaining the results here presented. We remark that our numerical model corresponds to an inviscid constitutive response and quasi-static loading conditions. It means that there are neither a constitutive (like a viscosity factor) nor a mass matrix 
terms regularizing the problem. The robustness of the Impl-Ex algorithm allow us to solve the ductile fracture problems as a sequence of linear ones. The error norm convergence rate of the Impl-Ex algorithm is similar to the implicit ones. The numerical solution of every time integration step by means of this procedure, in the linear kinematics context, requires only one iteration to converge. Therefore, every step needs only one stiffness matrix factorization.

(iii) The material instability condition in the stain localization direction, even when it is not the critical one, has been studied. Its relation with the large-scale-size void coalescence has been remarked.

The regularization of the GTN constitutive model is imperative. In a forthcoming paper, the author will be addressing this issue based on the strong discontinuity approach (Oliver et al., 1999, Oliver et al., 2002). In order to reach a successful implementation of this technique, the studies presented in this paper becomes mandatory.

\section{Acknowledgements}

Financial support from the Spanish Ministry of Science and Technology trough grant BIA2005-09250-C03-03 and from ANPCyT of Argentina through grant PICT-200534273, are gratefully acknowledged.

\section{Appendix A}

\section{A.1. Explicit form of the matrix Jacobian components in Eq. (34)}

Each term of the Jacobian tensor must be computed as follows:

$$
\begin{aligned}
J_{a a} & =\frac{\partial R_{a}}{\partial \Delta \varepsilon_{p}^{\mathrm{p}}}=\frac{1}{2 \sigma_{0}^{2}}\left[4 q+9 q_{1} q_{2}^{2} \kappa f^{*} \Delta \varepsilon_{q}^{\mathrm{p}} \cosh (\alpha)\right] ; \quad \alpha=-\frac{3}{2} \frac{q_{2} p}{\sigma_{0}} \\
J_{a b} & =\frac{\partial R_{a}}{\partial \Delta \varepsilon_{q}^{\mathrm{p}}}=\frac{1}{\sigma_{0}^{2}}\left[-6 \mu \Delta \varepsilon_{p}^{\mathrm{p}}+3 q_{1} q_{2} \sigma_{0} f^{*} \sinh (\alpha)\right] \\
J_{a c} & =\frac{\partial R_{a}}{\partial \bar{\varepsilon}}=-\frac{1}{2 \sigma_{0}^{3}}\left[8 q \Delta \varepsilon_{p}^{\mathrm{p}}+3 q_{1} q_{2} f^{*} \Delta \varepsilon_{q}^{\mathrm{p}}\left(3 q_{2} p \cosh (\alpha)+2 \sigma_{0} \sinh (\alpha)\right)\right] \frac{\partial \sigma_{0}}{\partial \bar{\varepsilon}} \\
J_{a d} & =\frac{\partial R_{a}}{\partial \Delta f}=\frac{3}{\sigma_{0}} q_{1} q_{2} \Delta \varepsilon_{q}^{\mathrm{p}} \sinh (\alpha) \frac{\partial f^{*}}{\partial f} \\
J_{b a}= & \frac{\partial R_{b}}{\partial \Delta \varepsilon_{p}^{\mathrm{p}}}=\frac{3}{\sigma_{0}} q_{1} q_{2} \kappa f^{*} \sinh (\alpha) \\
J_{b b}= & \frac{\partial R_{b}}{\partial \Delta \varepsilon_{q}^{\mathrm{p}}}=-\frac{6}{\sigma_{0}^{2}} q \mu \\
J_{b c}= & \frac{\partial R_{b}}{\partial \Delta \bar{\varepsilon}}=-\frac{1}{\sigma_{0}^{3}} \frac{\partial \sigma_{0}}{\partial \bar{\varepsilon}}\left[2 q^{2}+3 q_{1} q_{2} p \sigma_{0} f^{*} \sinh (\alpha)\right] \\
J_{b d}= & \frac{\partial R_{b}}{\partial \Delta f}=2 \frac{\partial f^{*}}{\partial f}\left[q_{1} \cosh (\alpha)-q_{3} f^{*}\right] \\
J_{c a}= & \frac{\partial R_{c}}{\partial \Delta \varepsilon_{p}^{\mathrm{p}}}=-\frac{\kappa \Delta \varepsilon_{p}^{\mathrm{p}}+p}{(1-f) \sigma_{0}}
\end{aligned}
$$




$$
\begin{aligned}
J_{c b} & =\frac{\partial R_{c}}{\partial \Delta \varepsilon_{q}^{\mathrm{p}}}=\frac{-3 \mu \Delta \varepsilon_{q}^{\mathrm{p}}+q}{(1-f) \sigma_{0}} \\
J_{c c} & =\frac{\partial R_{c}}{\partial \Delta \bar{\varepsilon}}=\frac{\left(p \Delta \varepsilon_{p}^{\mathrm{p}}-q \Delta \varepsilon_{q}^{\mathrm{p}}\right) \frac{\partial \sigma_{0}}{\partial \bar{\varepsilon}}-\sigma_{0}^{2}(1-f)}{(1-f) \sigma_{0}^{2}} \\
J_{c d} & =\frac{\partial R_{c}}{\partial \Delta f}=\frac{\left(p \Delta \varepsilon_{p}^{\mathrm{p}}-q \Delta \varepsilon_{q}^{\mathrm{p}}\right)}{(1-f)^{2} \sigma_{0}} \\
J_{d a} & =\frac{\partial R_{d}}{\partial \Delta \varepsilon_{p}^{\mathrm{p}}}=(1-f) \\
J_{d b} & =\frac{\partial R_{d}}{\partial \Delta \varepsilon_{q}^{\mathrm{p}}}=0 \\
J_{d c} & =\frac{\partial R_{d}}{\partial \Delta \bar{\varepsilon}}=\Delta \bar{\varepsilon} \frac{\partial \mathscr{A}_{m}}{\partial \Delta \bar{\varepsilon}}+\mathscr{A}_{m} \\
J_{d d} & =\frac{\partial R_{d}}{\partial \Delta f}=-\left(\Delta \varepsilon_{q}^{\mathrm{p}}+1\right) \\
\boldsymbol{A}_{11} & =\left[\begin{array}{ll}
J_{a a} & J_{a b} \\
J_{b a} & J_{b b}
\end{array}\right] \\
\boldsymbol{A}_{12} & =\left[\begin{array}{ll}
J_{a c} & J_{a d} \\
J_{b c} & J_{b d}
\end{array}\right] \\
\boldsymbol{A}_{21} & =\left[\begin{array}{ll}
J_{c a} & J_{c b} \\
J_{d a} & J_{d b}
\end{array}\right] \\
\boldsymbol{A}_{22} & =\left[\begin{array}{ll}
J_{c c} & J_{c d} \\
J_{d c} & J_{d d}
\end{array}\right]
\end{aligned}
$$

\section{A.2. Useful expressions}

In this appendix we write the final expressions for some variables defined and used in previous sections of the paper:

$$
\begin{aligned}
& \boldsymbol{M}=\frac{\partial \phi}{\partial \boldsymbol{\sigma}}=\frac{q_{1} q_{2} f^{*}}{\sigma_{0}} \sinh (\alpha) \mathbb{1}+\frac{3}{\sigma_{0}^{2}} \boldsymbol{S} ; \quad \alpha=-\frac{3}{2} \frac{q_{2} p}{\sigma_{0}} \\
& \operatorname{tr}(\boldsymbol{M})=(\mathbb{1}: \boldsymbol{M})=\frac{3 q_{1} q_{2} f^{*}}{\sigma_{0}} \sinh (\alpha) \\
& \boldsymbol{C}^{\mathrm{e}}=\lambda \mathbb{1} \otimes \mathbb{1}+2 \mu \llbracket=\kappa \mathbb{1} \otimes \mathbb{1}+2 \mu\left[\mathbb{\square}-\frac{1}{3} \mathbb{1} \otimes \mathbb{1}\right]
\end{aligned}
$$

where $\mu$ and $\lambda$ are the elastic Lame's parameters, and $\kappa$ the bulk modulus.

$$
\begin{aligned}
& \mathbb{1}=\delta_{i j}\left(\boldsymbol{e}_{i} \otimes \boldsymbol{e}_{j}\right) ; \quad \mathbb{\square}=\frac{1}{2}\left(\delta_{i k} \delta_{j l}+\delta_{i l} \delta_{j k}\right)\left(\boldsymbol{e}_{i} \otimes \boldsymbol{e}_{j} \otimes \boldsymbol{e}_{k} \otimes \boldsymbol{e}_{l}\right) \\
& \delta_{i j}: \text { Kronecker's Delta; } \quad \delta_{i j}= \begin{cases}1 & \text { if } i=j \\
0 & \text { if } i \neq j\end{cases} \\
& \boldsymbol{Q}^{\mathrm{e}}=\boldsymbol{\eta} \cdot \boldsymbol{C}^{\mathrm{e}} \cdot \boldsymbol{\eta}=\mu \mathbb{1}+(\mu+\lambda) \boldsymbol{\eta} \otimes \boldsymbol{\eta}
\end{aligned}
$$




$$
\begin{aligned}
& \boldsymbol{P}=\left(\boldsymbol{C}^{\mathrm{e}}: \boldsymbol{M}\right)=\kappa \operatorname{tr}(\boldsymbol{M}) 1+\frac{6 \mu}{\sigma_{0}^{2}} \boldsymbol{S} \\
& \left(\boldsymbol{M}: \boldsymbol{C}^{\mathrm{e}}: \boldsymbol{M}\right)=\frac{18 \mu}{\sigma_{0}^{4}}\|\boldsymbol{S}\|^{2}+\kappa[\operatorname{tr}(\boldsymbol{M})]^{2} \\
& (\boldsymbol{\sigma}: \boldsymbol{M})=-p \operatorname{tr}(\boldsymbol{M})+\frac{3}{\sigma_{0}^{2}}(\boldsymbol{S}: \boldsymbol{S}) \\
& \mathrm{N}=\frac{\partial \phi}{\partial f}=\frac{\partial \phi}{\partial f^{*}} \frac{\partial f^{*}}{\partial f}=\left[2 q_{1} \cosh (\alpha)-2 q_{3} f^{*}\right] \frac{\partial f^{*}}{\partial f} \\
& \mathrm{R}=\frac{\partial \phi}{\partial \bar{\varepsilon}}=\frac{\partial \phi}{\partial \sigma_{0}} \frac{\partial \sigma_{0}}{\partial \bar{\varepsilon}}=\left[-\frac{2 q^{2}}{\sigma_{0}^{3}}+\frac{p}{\sigma_{0}} \operatorname{tr}(\boldsymbol{M})\right] \underbrace{\frac{\partial \sigma_{0}}{\partial \bar{\varepsilon}}}_{H}
\end{aligned}
$$

\section{References}

Aravas, N., 1987. On the numerical integration of a class of pressure-dependent plasticity models. Int. J. Numer Methods Eng. 24, 1395-1416.

Belytschko, T., Chen, H., Xu, J., Zi, G., 2003. Dynamic crack propagation based on loss of hyperbolicity and a new discontinuous enrichment. Int. J. Numer. Methods Eng. 58, 1873-1905.

Besson, J., Steglich, D., Brocks, W., 2001. Modeling of crack growth in round bar and plane strain specimens. Int. J. Solids Struct. 38, 8258-8284.

Besson, J., Steglich, D., Brocks, W., 2003. Modeling of plane strain ductile rupture. Int. J. Plasticity, 1517-1541.

Betegon, C., del Coz, J., Peñuelas, I., 2006. Implicit integration procedure for viscoplastic Gurson material. Comput. Methods Appl. Mech. Eng. 195, 6146-6157.

Chu, C., Needleman, A., 1980. Void nucleation effects in biaxially stretched sheets. J. Eng. Mater. Technol. 102, 249-256.

Gao, X., Faleskog, J., Shih, C., 1998. Cell model for nonlinear fracture analysis. Part II: fracture process calibration and verification. Int. J. Fract. 89, 375-398.

Gurson, A., 1975. Plastic flow and fracture behavior of ductile materials incorporating void nucleation, growth and coalescence. Ph.D. thesis, Brown University.

Gurson, A., 1977. Continuum of ductile rupture by void nucleation and growth - Part I: Yield criteria and flow rules for porous ductile media. J. Eng. Mater. Technol. ASME 99, 2-15.

Hill, R., 1950. The Mathematical Theory of Plasticity. Oxford University Press, Oxford.

Kojic, M., Vlastelica, I., Zivkovic, M., 2002. Implicit stress integration procedure for large strain deformation of Gurson model. Int. J. Numer. Methods Eng. 53, 2701-2720.

Needleman, A., Tvergaard, V., 1987. An analysis of ductile rupture modes at crack tip. J. Mech. Phys. Solids 35, $151-183$.

Oliver, J., Huespe, A., 2004. Theoretical and computational issues in modelling material failure in strong discontinuity scenarios. Comput. Methods Appl. Mech. Eng. 193, 2987-3014.

Oliver, J., Cervera, M., Manzoli, O., 1999. Strong discontinuities and continuum plasticity models: the strong discontinuity approach. Int. J. Plasticity 15 (3), 319-351.

Oliver, J., Huespe, A.E., Pulido, M.D.G., Chaves, E., 2002. From continuum mechanics to fracture mechanics: the strong discontinuity approach. Eng. Fract. Mech. 69, 113-136.

Oliver, J., Huespe, A., Blanco, S., Linero, D., 2005. Stability and robustness issues in numerical modeling of material failure with the strong discontinuity approach. Comput. Methods Appl. Mech. Eng. 195 (52), $7093-$ 7114.

Ottosen, N., Runesson, K., 1991. Properties of discontinuous bifurcation solutions in elasto-plasticity. Int. J. Solids Struct. 27 (4), 401-421.

Pardoen, T., Hachez, F., Marchioni, B., Blyth, P.H., Atkins, A., 2004. Mode I fracture of sheet metals. J. Mech. Phys. Solids 52, 423-452.

Rice, J., 1976. The localization of plastic deformation. In: Koiter, W. (Ed.), Theoretical and Applied Mechanics, 14th IUTAM Congress. North-Holland, Amsterdam, pp. 207-220. 
Rice, J., Rudnicki, J., 1980. A note on some features of the theory of localization of deformation. Int. J. Solids Struct. 16, 597-605.

Rudnicki, J., Rice, J., 1975. Condition for the localization of deformations pressure sensitive dilatant materials. J. Mech. Phys. Solids 23, 371-394.

Simo, J., Hughes, T., 1998. Computational Inelasticity. Springer-Verlag.

Thomason, P., 1985. A three-dimensional model for ductile fracture by the growth and coalescence of microvoids. Acta Metall. 33, 1087-1095.

Thomason, P., 1998. A view on ductile-fracture modelling. Fatig. Fract. Eng. Mater. Struct. 21, 1105-1122.

Tvergaard, V., 1981. Influence of voids on shear band instabilities under plane strain conditions. Int. J. Fract. 17, 389-407.

Tvergaard, V., 1982. On localization in ductile materials containing spherical voids. Int. J. Fract. 18, 237-252.

Tvergaard, V., 1990. Material failure by void growth to coalescence. Adv. Appl. Mech. 27, 83-151.

Tvergaard, V., Hutchinson, J., 1992. The relation between crack growth resistance and fracture process parameters in elasto-plastic solids. J. Mech. Phys. Solids 40, 1377-1397.

Tvergaard, V., Hutchinson, J.W., 2002. Two mechanisms of ductile fracture: void by void growth versus multiple void interactions. Int. J. Solids Struct. 39, 3581-3597.

Tvergaard, V., Needleman, A., 1984. Analysis of the cup-cone fracture in a round tensile bar. Acta Metall. 32, $157-169$.

Tvergaard, V., Needleman, A., 2006. Three dimensional microstructural effects on plane strain ductile crack growth. Int. J. Solids Struct. 43, 6165-6179.

Willam, K., 2000. Constitutive models for engineering materials. Encyclopedia of Physical Science \& Technology, third ed. Academic Press, New York, pp. 603-633.

Xia, L., Shih, C., Hutchinson, J., 1995. Computational approach to ductile crack growth under large scale yielding conditions. J. Mech. Phys. Solids 43, 389-413.

Zhang, Z., 1995. On the accuracies of numerical integration algorithms for Gurson-based pressure-depended elastoplastic constitutive models. Comput. Methods Appl. Mech. Eng. 121, 29-44.

Zhang, Z., 1998. A complete Gurson model. In: Aliabadi, M. (Ed.), Nonlinear Fracture and Damage Mechanics. Computational Mechanics Publications. 